\title{
Nouvelles données sur les Asellidae épigés d'Extrême-Orient (Crustacea, Isopoda, Asellota)
}

\author{
Jean-Paul Henry \& Guy Magniez \\ Université de Bourgogne, Département de Biologie Animale, Écologie, 6 Bd. Gabriel, 21000 Dijon, \\ France
}

Keywords: Asellus (Asellus) levanidovorum n. sp.; gen. Asellus n. def.; Sakhalin and Kunashir Islands; SEM; structure of copulatory pleopods; subgen. Asellus, subgen. Arctasellus nov.

\begin{abstract}
Numerous samples of oculate Asellus from eastern Siberia and the Japanese Islands, one from southern China, and one from Alaska, have been studied. The epigean fresh waters of this large Asiatic region generally harbour populations identified as Asellus (Asellus) hilgendorfii Bovallius, 1886, or some of its geographical forms previously considered as species, subspecies, or not yet named. The "hilgendorfii" complex seems to possess the dimension of a superspecies.

A new pigmented-oculate species, Asellus (Asellus) levanidovorum is sympatric with $A$. (A.) hilgendorfii in the lake Bolon region (lower part of the Amur River basin) and is present also in the Sakhalin and Kunashir Islands. Its copulatory organ (endopodite of male 2nd pleopod) shows a large cannula and study with SEM allows us to understand the organizational pattern of this organ in all species belonging to the true genus Asellus, including the type-species Asellus (A.) aquaticus (Linnaeus, 1758) where the cannula is small and deeply hidden. Strong relationship between these species points to the existence of a subgenus Asellus inside the genus Asellus auct.

The three Arctic species, A. latifrons Birstein, 1947, A. alaskensis Bowman \& Holmquist, 1975 and $A$. birsteini Levanidov, 1976, represent a second subgenus, Arctasellus nov., inside the genus Asellus car. emend., characterized by their onisciform aspect, the reduction of the coxopodites of all pereiopods, and the structure of the different parts of the copulatory appendages, homologous with those of the subgenus Asellus, but simpler and exposed.

The Asellidae appear to be a heterogeneous family, having taken origin from several marine ancestors, and having colonized independently the fresh waters of several remote continental sites. These separate elements evolved independently in the North Pacific (Japan Sea region), Baikal, the Mediterranean and Paratethys, southwestern Atlantic Europe, and Atlantic North America.
\end{abstract}

All species of the far-eastern group (genera Asellus, Calasel- lus. Mesoasellus, Nipponasellus, Phreatoasellus, Sibirasellus, and Uenasellus) show a common general architecture of the copulatory appendages, with homologous elements ("Asellus pattern") in each of them. This multigeneric group represents a distinct unit inside the family Asellidae. The presence of its main member, Asellus (A.) aquaticus, in Europe is the result of a recent and long-range migration westward inside the natural area of the autochthonous Mediterranean forms.

\section{Résumé}

De nombreux prélèvements d'Asellus oculés provenant de Sibérie orientale ainsi que des îles japonaises plus un de Chine méridionale et un autre d'Alaska ont été examinés. Les eaux douces épigées de cette vaste région asiatique renferment essentiellement des populations déterminées comme Asellus hilgendorfii Bovallius, 1886 et ses variants géographiques antérieurement désignés comme espèces, sous-espèces ou non encore nommés. Le complexe "hilgendorfii" semble présenter la dimension taxinomique d'une super-espèce.

Une espèce pigmentée et oculée nouvelle: Asellus (Asellus) levanidovorum est sympatrique d'A. (A.) hilgendorfii dans la région du lac Bolon (bassin inférieur de l'Amour) et également présente à Sakhaline et Kunashir. L'organe copulateur du mâle d'A. (A.) levanidovorum n. sp. est muni d'une grande canule et son étude à l'aide du S.E.M. permet de préciser l'unité du plan d'organisation de l'endopodite du pléopode 2 mâle des véritables Asellus, en particulier de l'Asellus (A.) aquaticus (Linnaeus, 1758) d'Europe chez lequel cette canule est minuscule et masquée. L'étroite parenté des espèces précitées permet de mieux délimiter une des deux lignées évolutives (sous-genre Asellus) à l'intérieur du genre Asellus auct.

Les Asellus arctiques des espèces A. latifrons Birstein, 1947, A. alaskensis Bowman \& Holmquist, 1975 et $A$. birsteini Levanidov, 1976, constituent un second rameau évolutif du 
genre Asellus (sous-genre Arctasellus), caractérisé par le faciès oniscoïde, la réduction des coxopodites des péréiopodes et une structure de l'organe copulateur homologue de celle du sousgenre Asellus, mais plus simple et non protégée.

Les Asellidae apparaissent de plus en plus comme une famille hétérogène, dont les différents ancêtres marins ont colonisé les eaux douces, à des époques différentes et indépendamment, à partir de plusieurs centres de dispersion continentale géographiquement éloignés: Nord-Pacifique (zone de la Mer du Japon), Baikkal, Méditerranée et Paratéthys, Europe atlantique, Amérique du Nord atlantique.

Toutes les espèces du groupe extrême-oriental (genres Asellus, Calasellus, Mesoasellus, Nipponasellus, Phreatoasellus, Sibirasellus et Uenasellus) présentent une architecture génerale similaire de l'appareil copulateur, composé d'éléments homologues ("Asellus pattern"), ce qui est l'indice d'une origine commune, et constituent donc une unité évolutive originale au sein de la famille Asellidae. La présence du représentant le plus connu de ce groupe: Asellus (A.) aquaticus en Europe, dans l'aire naturelle du groupe méditerranéen autochtone, est la conséquence d'une migration récente à longue distance vers l'ouest.

\section{Généralités et origine du matériel}

A la suite des prospections effectuées en Sibérie orientale continentale et insulaire, à partir de 1958, par des chercheurs de l'Institut de Biologie et Pédologie de Vladivostok, des prospections du Dr. N.W. Broodbakker d'Amsterdam dans les îles japonaises (1986) et des récoltes de faune opérées en Chine par Mme et Mr. Lips de Lyon (1992), de nombreux échantillons d'Asellidae pigmentés et oculés d'Extrême-Orient ont pu être réunis et permettent de réaliser une révision de cette faune d'Isopodes. Le matériel se répartit ainsi:

Une collection de 34 tubes (notés ici L.1 à L.34), contenant des Asellides pigmentés et oculés, récoltés entre 1958 et 1988, de la région de Vladivostok et ses îles côtières (L.1 à 7), du lac Khanka (L.8), de Khabarovsk, du lac Bolon et du bassin inférieur de l'Amour (L.9 à 13), des îles Sakhaline (L.29 et 30) et Kunashir (L.32 et 33), des régions du Kamchatka (L.31), du Magadan (L.27 et 28), d'Anadyr, de la péninsule Chukotka (L.14 à 26) et enfin d'Alaska (L.34). Cette collection nous a été confiée par le Dr. I.M. Levanidova (Vladivostok) à la suite de la disparition du regretté Dr. Levanidov.

Une collection de 9 tubes (notés ici J.15, 25, 26, 37, 41, 44, 59, 133 et 135), contenant des Asellides pigmentés et oculés récoltés en 1986, au Japon: Honshu (J.15-44), île Okinawa (J.59), île Yoron-to (J.133 et 135), par le Dr. N.W. Broodbakker (Université d'Amsterdam). Ce matériel nous a été confié par le Professeur J.H. Stock (Amsterdam).
Un tube (noté ici C.1) contentant des Asellides oculés de la rivière souterraine de Xing'an (province de Guangxi, Chine); matériel récolté par $\mathbf{B}$. et $\mathbf{J}$. Lips de Lyon.

Tout ce matériel est inventorié dans les collections du Musée de Zoologie de l'Université d'Amsterdam (ZMA). Nous remercions très vivement toutes les personnes qui ont réalisé les collectes ou nous ont réservé le matériel pour la confiance qu'ils nous ont témoignée.

\section{Les Asellus du groupe "aquaticus-hilgendorfii" (sous-genre Asellus)}

\section{A. Le problème taxinomique d'Asellus hilgendorfii}

Le premier Asellide d'Extrême-Orient (Asellus sp.) a été signalé par Hilgendorf en 1874. La station originelle serait située près de la localité de Yedo à Hokkaido (cf. Matsumoto, 1963: 27). L'espèce fut désignée comme Asellus ? Hilgendorfii par Bovallius (1886: 13) et en 1893, Hilgendorf utilisa à son tour ce binôme pour désigner d'autres Aselles épigés du Japon; il fut suivi dans cette voie par Kawamura (1918). Par la suite, plusieurs auteurs: Tattersall (1921), pour des Aselles du lac Biwa, Derjavin en 1930, pour des Aselles de l'ExtrêmeOrient soviétique et Shen en 1936, pour des Aselles de Chine, considèrent leurs spécimens comme appartenant à l'espèce européenne Asellus (Asellus) aquaticus (Linnaeus, 1758). Négligeant. tous les travaux antérieurs, Nicholls (1929) désigna les Aselles japonais de la région du lac Chuzenji (près de Nikko, Honshu), sous le nom d'Asellus nipponensis, dont Uéno (1938) sépara une sous-espèce méridionale: $A$. n. ryukyuensis. En 1932, Birstein mit en doute, à juste titre, la présence d'Asellus aquaticus en Asie orientale (cette question n'est toujours pas réglée, puisque Wei, en 1970, signale encore la présence, à la fois d'A. aquaticus et d'A. hilgendorfii dans les environs de Hangchow à $150 \mathrm{~km}$ au S.O. de Shanghai).

En 1947, Birstein décrivit Asellus hilgendorfii martinovi des marécages du delta de la Léna et en 1951, il considéra tous les Aselles cités plus haut comme appartenant à l'unique espèce Asellus hilgendorfii. Cette position fut reprise par Matsumoto (1963) qui cita de nombreuses stations de toutes les îles japonaises. Ainsi, Birstein (1951) et Matsumoto 
(1963) purent donner la répartition suivante pour A. h. hilgendorfii: archipel nippon, Chine et Mandchourie, Corée, Extrême-Orient soviétique (bassins de l'Amour, de l'Oussouri, du Suifun), sauf le Kamchatka; île Sakhaline et îles Shantar. Une carte, conforme aux données précédentes et montrant la répartition des Asellides en ExtrêmeOrient, fut publiée par Bowman \& Holmquist en 1975. Finalement, en 1980, Levanidov décrivit des Aselles provenant de la Sibérie orientale arcrique (région de l'Anadyr et péninsule Chukotka) sous les noms d'Asellus (A.) beringianus et $A$. (A.) tshaunensis. Ces formes sont extrêmement voisines de la grande espèce polymorphe $A$. (A.) hilgendorfii des auteurs.

\section{B. Les observations}

Dans sa faune de l'URSS, Birstein (1951) indique: ". . . A comparison of Japanese Asellus specimens collected by Derzhavin on Hokkaido with Asellus from Amur and Sakhalin proves that they all belong to one and the same species...". Comme cette aire encadre la localité-type d'Hilgendorf (Hokkaido), nous avons pensé y trouver les Asellus hilgendorfii les plus typiques et examiné en priorité les prélèvements provenant du bassin inférieur de l'Amour (lac Bolon, $\mathrm{n}^{\circ}$ L.9), de Sakhaline ( $\mathrm{n}^{\circ}$ L.29, L. 30) et de l'îile Kunashir ( $n^{\circ}$ L.32, L.33).

A notre surprise, nous avons constaté que le lot L. 9 renfermait deux types morphologiques d'indivus, parfaitement différenciables les uns des autres (et aucun individu de type intermédiaire). Dans cette localité, il y a donc cohabitation de deux espèces épigées sympatriques indépendantes. L'un des lots correspond à l'Asellus (Asellus) hilgendorfii des auteurs, dont il sera question plus loin (lot L.9b); l'autre (lot L.9a) à un Asellide plus robuste et plus fortement pigmenté dont les pléopodes 2 mâles, même s'ils ont l'aspect général indiqué dans les schémas publiés précédemment pour les espèces asiatiques du genre Asellus, présentent un endopodite (organe copulateur) muni d'une volumineuse canule, faisant largement saillie hors de la crypte sternale de l'endopodite. Par suite de sa longueur, cette canule (au moins sa moitié distale) est par- faitement visible en microscopie optique (Figs. 11, 12), ce qui n'est pas le cas pour $A$. hilgendorfii. Rappelons que l'existence d'une canule chez $A$. hilgendorfii (Pl. I, A) et les espèces stygobies apparentées n'a pu être montrée que grâce à la microscopie électronique à balayage (SEM) (Henry \& Magniez, 1991). En effet, dans les préparations de pléopode 2 mâle de cette espèce pour la microscopie optique, l'organe se place naturellement parallèlement à la lame et à la lamelle de verre, face sternale vers le haut; dans cette situation, la petite canule se trouve exactement en dessous de la bordure écailleuse du lobe antérieur et ne déborde pas les limites de la crypte délimitée par ce lobe antérieur et le capitulum. De ce fait, elle est pratiquement indécelable, à moins que l'on ne soupçonne à l'avance son existence, ce qui explique son absence dans les schémas des auteurs antérieurs. Chez A. levanidovorum $\mathbf{n}$. sp., la réalité de son existence est facilement confirmée par le SEM (PI. I, B-E). Cette structure se retrouve identique chez les individus de Sakhaline (L.29 et L.30, Fig. 13) et de Kunashir (L.32 et L.33, Fig. 14). Il s'agit donc là d'une espèce nouvelle présentant une aire de répartition limitée; elle est décrite ci-dessous et dédiée à nos collègues, le regretté Dr. Levanidov et le Dr. Levanidova.

\section{Asellus (Asellus) levanidovorum n. sp. (Figs. 1-2, 4-18, 27, Pl. I, B-E)}

Matériel examiné. - Numéro de collection pour tout ce matériel asellidien: ZMA Isop. 200991.

Localités et lots capturés: L.9a, "Lower part of Amur river, tributaries of Bolon lake, 12-14/05/1958, Levanidova coll.": une série de mâles adultes de 11,4 (holotype); 10,$4 ; 10,4 ; 10,0$; 9,$1 ; 8,5 ; 8,0 ; 7,9 ; 7,8 ; 7,7 ; 7,3 ; 6,2 \mathrm{~mm}$, plus la moitié antérieure d'un autre mâle; une série de femelles adultes à oostégites de repos génital de 9,0 (allotype); 9,$0 ; 8,5 ; 7,8 ; 7,4 ; 7,4 ; 7,3 ; 7,1 ; 6,5$; $6,2 \mathrm{~mm}$ et une femelle ovigère de $7,3 \mathrm{~mm}$ avec 22 oeufs jauneorangés dans le marsupium (la portée semble incomplète), plus la partie antérieure de 5 femelles adultes à oostégites réduits et 3 parties postérieures.

L.29, "Sakhalin island, spring in the vicinity of Neftegorsk town, 05/07/1985, Makarchenko coll.": un grand mâle adulte de $11,0 \mathrm{~mm}$.

L.30, "Sakhalin isl. Ado-Timova village, Blagodatnyi spring, 10/09/1985, Makarchenko coll.": un mâle adulte de 7,6 mm.

L.32, "Kunashir isl., Sernovodsk town, Pescharaya river, 26/05/1976, Vshivkova coll.": un mâle immature de 3,6 mm (le 


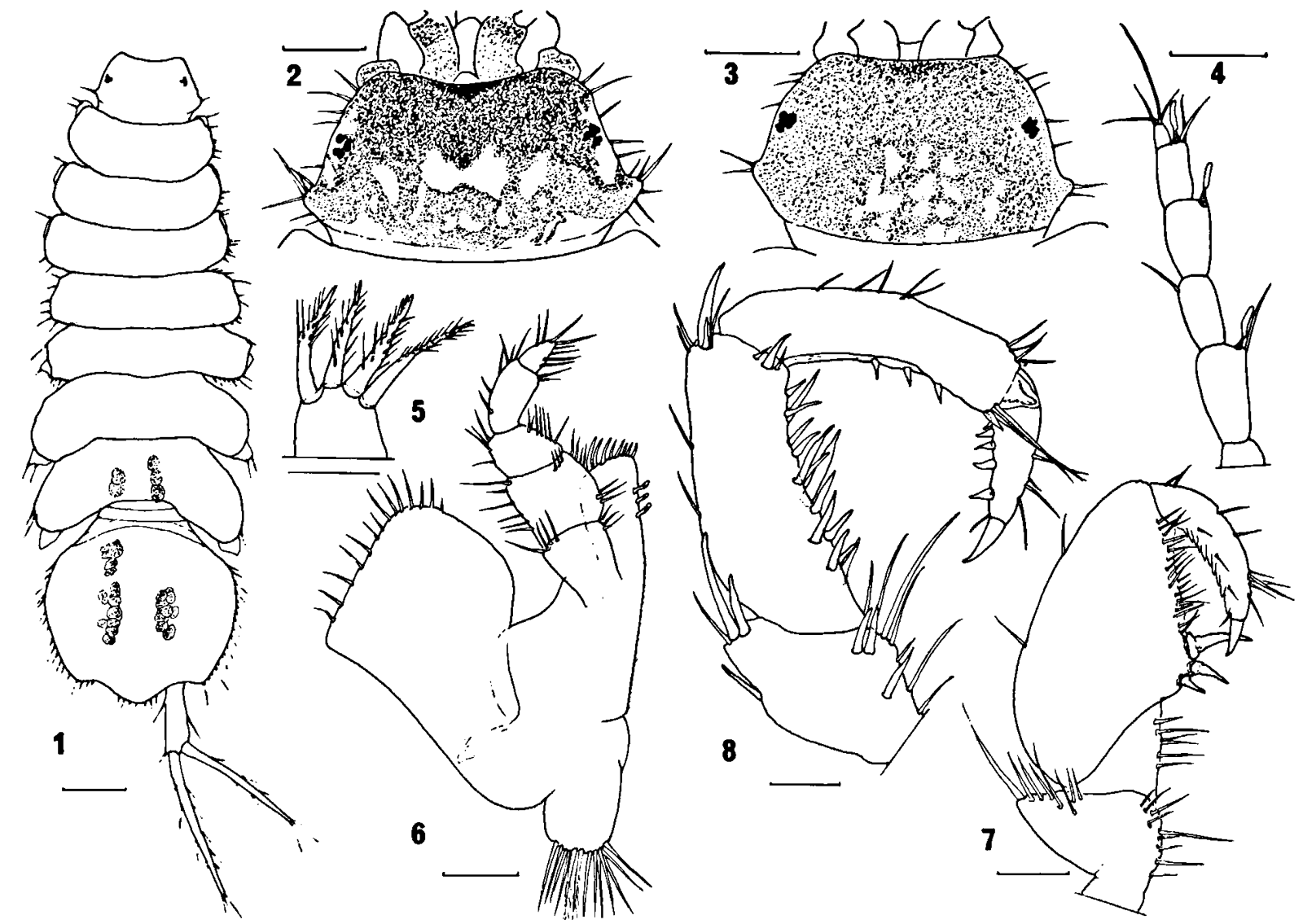

Figs. 1-8. Asellus (Asellus) levanidovorum n. sp.: 1, habitus d'un mâle de 10,4 mm, du lac Bolon, chromatophores non figurés, échelle $=1 \mathrm{~mm} ; 2$, céphalon du même individu, é $=0,5 \mathrm{~mm} ; 3$, céphalon d'un mâle d'A. (Asellus) hilgendorfii de la même localité, à titre de comparaison avec le précédent, é $=0,5 \mathrm{~mm} ; 4$, extrémité d'une antennule du mâle de $10,4 \mathrm{~mm}, \dot{e}=100 \mu \mathrm{m} ; 5$, lame interne d'une maxillule de la femelle ovigère de $7,3 \mathrm{~mm}$ du lac Bolon, é $=60 \mu \mathrm{m} ; 6$, maxillipède de la même, é $=100 \mu \mathrm{m} ; 7$, péréiopode 1 du mâle de $10,4 \mathrm{~mm}$, é $=300 \mu \mathrm{m} ; 8$, péréiopode 4 du même individu, é $=200 \mu \mathrm{m}$.

tube contient en outre de nombreux Sphaeromatidae pigmentés et oculés: ZMA Isop. 201 332).

L.33, "Kunashir isl., Pescharaya river, 26/05/1976, Vshivkova coll.": un mâle adulte de 7,1 mm et une femelle adulte de $5,5 \mathrm{~mm}$ à oostégites de repos génital.

Définition. - Asellus de forte taille; habitus semblable à celui d' $A$. hilgendorfii, mais pigmentation céphalique plus uniforme et plus intense, en particulier pour la moitié antérieure. Endopodite des pléopodes 2 mâles avec une canule de grande taille faisant largement saillie hors de l'organe.

Description. - Corps (Fig. 1) très robuste et bien sclérifié. La taille dépasse $11 \mathrm{~mm}$ pour les plus grands mâles. Dans la population du lac Bolon, la taille moyenne de la nouvelle espèce dépasse significativement celle des Asellus (A.) hilgendorfii qui cohabitent avec elle (taille maximale des mâles: $10,4 \mathrm{~mm}$ au lieu de $7,8 \mathrm{~mm}$, taille moyenne: $8,71 \mathrm{au}$ lieu de $5,91 \mathrm{~mm}$; taille maximale des femelles adultes: 9,0 au lieu de $6,5 \mathrm{~mm}$, taille moyenne: 7,60 au lieu de $5,12 \mathrm{~mm}$ ). Aspect général très semblable à celui d' $A$. hilgendorfii Bovallius, 1886 ou d' $A$. aquaticus (Linnaeus, 1758).

Céphalon (Fig. 2) à pigmentation dorsale très caractéristique, la coloration de la moitié antérieure étant très intense et celle de la moitié postérieure plus diffuse tandis que chaque oeil se trouve dans une plage très claire. Chez $A$. (A.) hilgendorfii, la 
pigmentation générale du céphalon (Fig. 3) est moins intense et plus uniforme, avec seulement de petites marbrures blanches dispersées. C'est cette observation qui a attiré en premier lieu l'attention sur le caractère hétérogène des prélèvements du lac Bolon et entraîné l'examen de chaque individu du lot.

Yeux (Fig. 2) formés de 3 ommatidies légèrement dissociées, très fortement pigmentés.

Sétation générale très fournie. Comme c'est la règle chez les Asellidae sur la plupart des organes, le nombre de soies est lié à la croissance et plus élevé chez les plus grands individus ayant subi davantage de mues.

Péréion: comme chez les autres Asellus du type "aquaticus-hilgendorfii" les coxopodites sont bien développés et les marges des épimères visibles dorsalement. La plupart des femelles du lac Bolon montrent par transparence les deux ovaires remplis d'ovocytes jaune-orangés ayant atteint leur taille définitive: elles sont donc sur le point d'effectuer leur mue parturielle et de pondre. Une seule femelle du lot a déjà effectué cette ponte et l'état de développement des embryons du marsupium indique qu'elle était très récente. La capture étant intervenue entre le 12 et le 14 mai, on peut en déduire que la reproduction doit présenter un maximum en juin dans cette station.

Pléon: les pléonites 1 et 2 sont très petits et forment un pédoncule largement recouvert par la marge postérieure du dernier péréionite. Pléotelson (Fig. 1) subcordiforme avec des sutures correspondant aux limites des pléonites 3-4 et $4-5$ bien visibles à l'avant. Pointe caudale bien marquée. Le grand développement des muscles uropodiaux dans ce pléotelson montre qu'une grande partie en revient au pléonite 6 . De part et d'autre de l'intestin postérieur existe un alignement longitudinal de vésicules internes à paroi très fortement pigmentée de brun et visibles par transparence (vraisemblablement des groupes de cellules de Zencker). Ces formations sont absentes ou incolores chez $A$. ( $A$.) hilgendorfii, dont le pléotelson apparaît beaucoup plus clair.

Appendices céphaliques: antennules (Fig. 4) plus lorigues que le céphalon, avec hampe de 4 et fouet de 13 articles dont les 9 ème, 11 ème et 12 ème portent une lame olfactive de $45 \mu \mathrm{m}$ de long chez le mâle de $10,4 \mathrm{~mm}$. Le fouet de la femelle de 7,3 mm ne compte que 9 articles, mais également 3 aesthétasques. Antennes relativement longues $(5,8 \mathrm{~mm}$ pour la femelle de $7,3 \mathrm{~mm}$ ), avec fouet pouvant compter jusqu'à 65 articles chez les plus grands individus intacts.

Mandibules typiques, très semblables à celles d'A. hilgendorfii. Maxillules avec lame interne portant 4 fortes tiges ramifiées sur sa marge distale (Fig. 5). Rappelons que ce nombre est caractéristique des véritables "Asellus", alors que le nombre de base de 5 tiges se rencontre chez la plupart des genres d'Asellidae et chez d'autres Aselloïdes ou Asellota. Maxilles trilobées sans particularité notable, très semblables à celles d' $A$. hilgendorfii.

Maxillipèdes typiques, avec un rétinacle de 3-5 crochets et un palpe sétigère de 5 articles. Chez la femelle ovigère, l'oostégite temporaire est représenté par un petit lobe (Wasserstrudelapparat) portant 25 à 28 tiges, les unes lisses, les autres plumeuses, toutes à direction caudale (Fig. 6).

Péréiopodes: péréiopodes 1 du mâle (Fig. 7) avec propodite fortement renflé, la partie basale de sa marge palmaire munie d'une forte expansion armée de 3 épines ensiformes (structure caractéristique des véritables "Asellus"); le reste de cette marge est subrectiligne et muni d'une vingtaine de tiges lisses. Marge palmaire du dactylopodite avec une dizaine de lames à bords lisses. Dactylopodites des péréiopodes 2-7 comptant jusqu'à 5 épines sternales, en particulier sur les appendices postérieurs; la plus proche de la base de l'ongle, qui est toujours la première à apparaître, étant toujours la plus longue. Péréiopode 4 du mâle (Fig. 8) légèrement raccourci et conformé en "crochet nuptial". Papilles génitales insérées sur le sternum du dernier péréionite, près de la marge interne du coxopodite correspondant.

Pléopodes 1 du mâle (Fig. 10) indépendants. Protopodite subquadrangulaire avec un rétinacle de 3-4 crochets sur la marge interne. Exopodite très régulièrement ovalaire, avec une vingtaine de très longues soies régulièrement espacées sur la marge distale et externe. Cette rangée est doublée de courtes soies lisses submarginales-sternales près de la marge distale et de soies identiques submargi- 


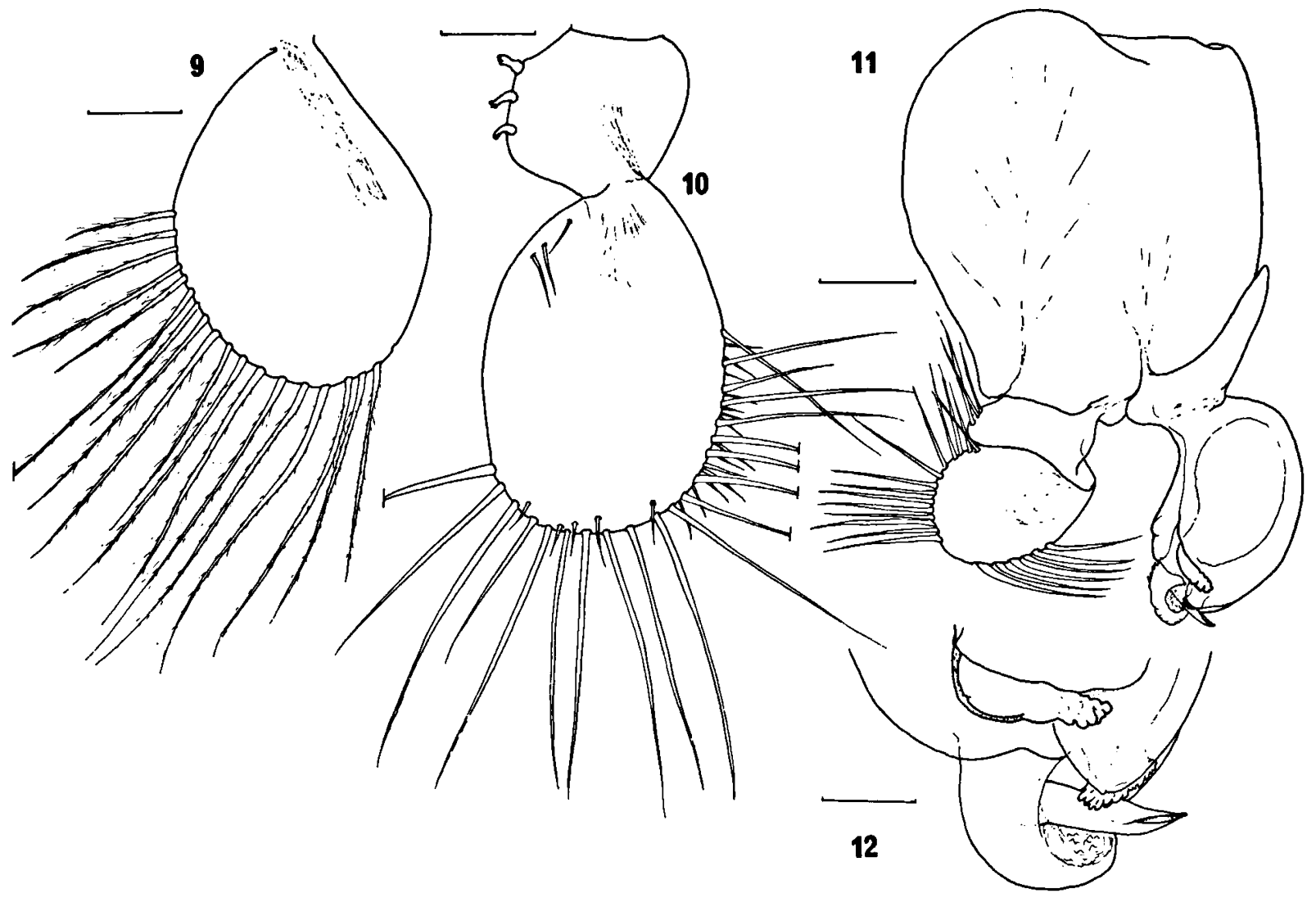

Figs. 9-12. Asellus (Asellus) levanidovorum n. sp.: 9, pléopode 2 droit, face sternale, de la femelle ovigère, échelle $=200 \mu \mathrm{m} ; 10$, pléopode 1 gauche, face sternale, du mâle de $10,4 \mathrm{~mm}$, é $=200 \mu \mathrm{m} ; 11$, pléopode 2 gauche, face sternale, du même, é $=100 \mu \mathrm{m} ; 12$, portion distale, face sternale, de l'endopodite du pléopode précédent, le processus cylindriformis et la longue canule sont bien distincts, $\hat{e}=50 \mu \mathrm{m}$.

nales-tergales au niveau de la marge externe. Ces appendices sont donc très similaires à ceux d' $A$. hilgendorfii, alors que ceux d'A. aquaticus présentent une différenciation spécifique de la marge externe de l'exopodite.

Pléopodes 2 du mâle (Figs. 11-12): protopodite subtrapézoïdal, un peu plus long que large. Sa cavité renferme une musculature extrêmement puissante, en particulier les muscles moteurs de l'exopodite. Article basal de l'exopodite court et cupuliforme, avec 2-3 tiges lisses sur sa marge externe. Article distal subovalaire, plus large que le précédent, présentant un lobe tergal (= catch lobe) très développé (structure caractéristique du genre Asellus et des genres apparentés) et une puissante musculature. Marge portant de nombreuses soies, dont un faisceau de fortes tiges parallèles, implantées sur la marge distale-interne et dirigées vers l'endopodite; les tiges des marges externe et distale-externe sont disposées en éventail. Endopodite d'aspect général similaire à celui d' $A$. hilgendorfii (Figs. 11 et 12) avec partie basale contenant un muscle endopodial et portant une très puissante apophyse basale (= basal spur ou processus calcariformis) présentant un sillon articulaire transversal, comme celui d' $A$. aquaticus ou d' $A$. hilgendorfii; sillon peu apparent en microscopie optique, mais parfaitement net avec le SEM. Partie renflée de l'endopodite ovoïde vésiculeuse, avec sa marge distaleexterne dilatée; orifice spermatique afférent (= fissure), très oblique et muni d'un volet membraneux prolongé par un processus digitiforme long et mamelonné comme chez $A$. aquaticus et $\boldsymbol{A}$. hilgendorfii (= labial spur ou processus cylindriformis); 
lobe antérieur légèrement spatulé, bien distinct et portant de grandes écailles digitées sur sa face distale; capitulum court et épais, à marge régulièrement convexe, avec quelques spinules proximalesexternes, sa concavité délimite une crypte où est insérée une volumineuse canule membraneuse conique dont seule la moitié distale fait saillie au delà des contours de l'organe. La base de cette canule est protégée par une membrane squamifère très développée, garnie de larges écailles à marge multidigitée et à disposition tégulaire (ces écailles sont nombreuses et plus larges, avec davantage de digitations; ces dernières moins longues que celles d' $\boldsymbol{A}$. hilgendorfii); la partie distale de cette canule est fissurée, latéralement, ce qui constitue l'orifice spermatique efférent.

Pléopodes 2 de la femelle (Fig. 9): subovalaires avec marge proximale-interne presque rectiligne. Les marges internes des deux appendices se recouvrent légèrement en position de repos. Une série de 19 longues tiges, toutes plumeuses, garnit les marges externe et distale.

Pléopodes 3 (Fig. 15): exopodite subovalaire, divisé en 2 articles par une suture oblique. Une série continue d'environ $\mathbf{4 0}$ soies garnit toute la marge externe et distale; les soies externes sont courtes et lisses, les distales plus longues et plumeuses.

Pléopodes 4 (Fig. 16): exopodite ovalaire très semblable à celui des autres Asellus épigés. L'aire charnue respiratoire est séparée du corps de l'exopodite par une ligne articulaire double, formant une lunule caractéristique. Une quinzaine de soies lisses sont insérées sur la marge proximale externe, par ailleurs finement ciliée. Endopodite subcirculaire charnu et respiratoire.

Pléopodes 5 (Fig. 17): exopodite ovalaire avec 7 soies lisses sur la marge proximale-externe et une aire charnue distale subquadrangulaire délimitée par une ligne double en croissant plus fermé que celui de l'exopodite 4. Endopodite ovalaire et charnu.

Uropodes (Fig. 1): plus long que le pléotelson, exopodite légèrement plus court que l'endopodite.

Affinités. - La nouvelle espèce montre tous les caractères généraux propres au genre Asellus sensu stricto, et au sein de celui-ci, au groupe d'espèces

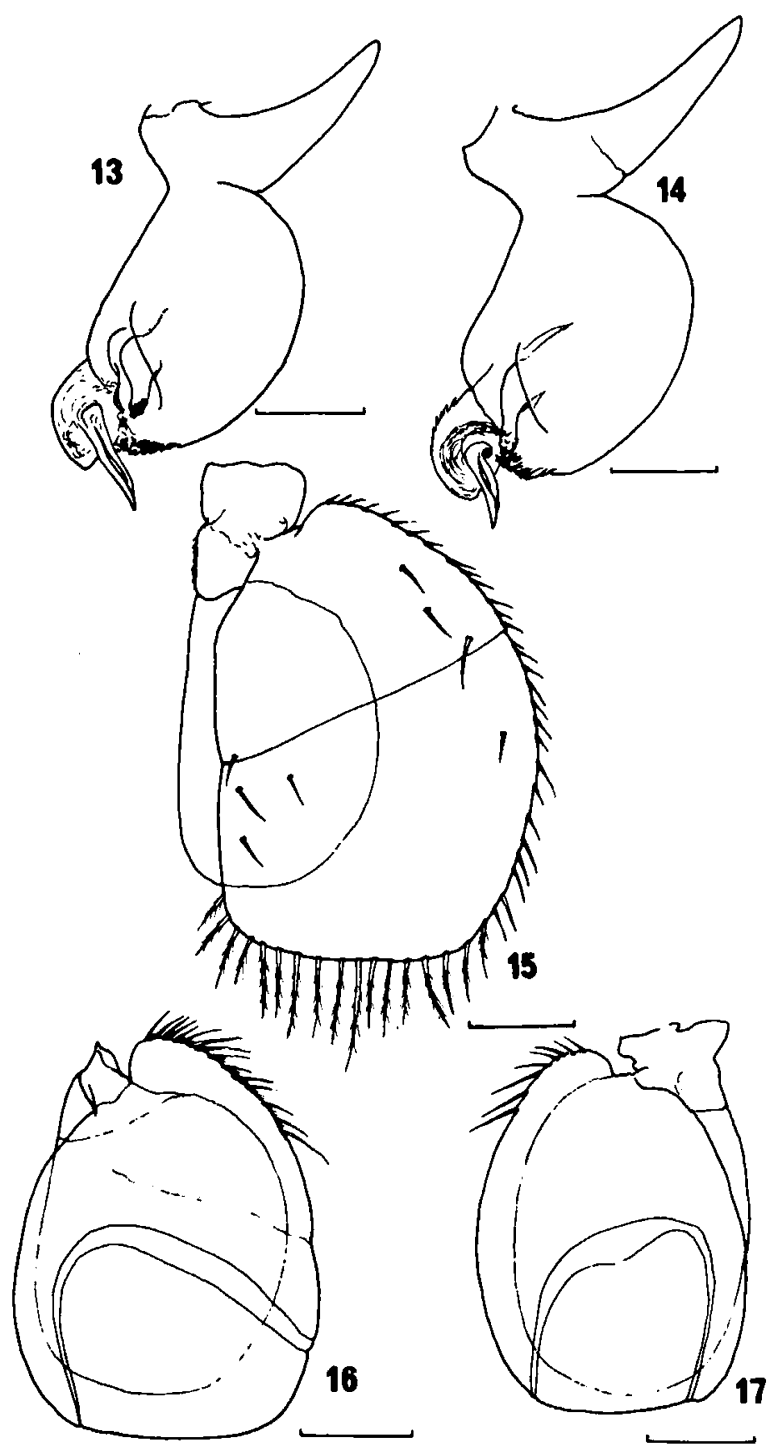

Figs. 13-17. Asellus (Asellus) levanidovorum n. sp.: 13, face sternale de l'endopodite du pléopode 2 mâle d'un exemplaire de Sakhaline, échelle $=100 \mu \mathrm{m} ; 14$, face sternale de l'endopodite du pléopode 2 mâle d'un exemplaire de Kunashir, é $=100 \mu \mathrm{m}$; 15 , pléopode 3 gauche, face sternale, du mâle de $10,4 \mathrm{~mm}$ du lac Bolon, é $=300 \mu \mathrm{m} ; 16$, pléopode 4 gauche, face sternale, du même, é $=300 \mu \mathrm{m} ; 17$, pléopode 5 gauche, face sternale, $\hat{e}=300 \mu \mathrm{m}$.

"aquaticus-hilgendorfii". La plupart de ses caractères morphologiques somatiques l'apparentent très étroitement à l'espèce polymorphe $A$. hilgendorfii des auteurs. Cependant, deux faits militent en faveur de son indépendance spécifique: (1) la différence considérable de structure de la partie ter- 


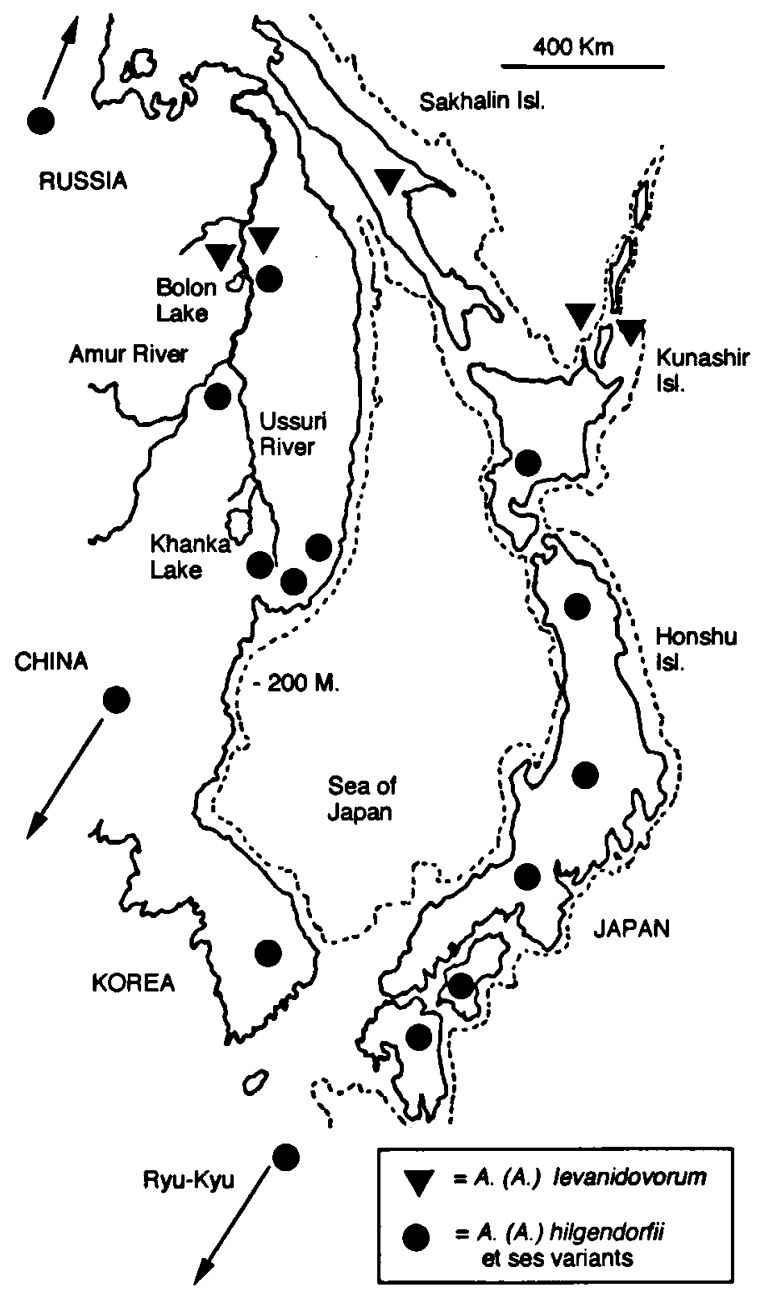

Fig. 18. Carte de répartition d'A. (A.) levanidovorum n. sp. et d'A. (A.) hilgendorfii (partiellement).

minale de l'organe copulateur mâle, muni d'une canule de grande taille chez $A$. levanidovorum (ce caractère se montrant constant, aussi bien chez les individus continentaux du bassin inférieur de l'Amour que dans les populations insulaires de Sakhaline et Kunashir, Figs. 13 et 14), et (2) également la présence simultanée (sympatrie) des deux formes "hilgendorfii" et levanidovorum" dans la région du lac Bolon (bassin inférieur de l'Amour, prélèvements notés L.9) et ceci sans apparition de types intermédiaires. Les cohabitations d'espèces sont bien connues dans la faune d'Europe: Asellus aquaticus et Proasellus meridianus (Racovitza, 1919) (deux oculés ensemble); Proasellus cavaticus
(Leydig, 1871) et Proasellus walteri (Chappuis, 1948) (deux anophtalmes) par exemple et l'on sait qu'aucune hybridation ne s'y produit, ce qui est un signe de la totale indépendence génétique des couples d'espèces sympatriques. La relative endémicité d' $A$. levanidovorum par rapport à $A$. hilgendorfii et le fait que toutes les espèces stygobies connues du Japon et du Primorye paraissent dériver de ce même $A$. hilgendorfii donnent à penser que le type "hilgendorfii" est le plus proche de la souche de tout ce complexe évolutif et que la canule de type "levanidovorum" représente une structure ayant évolué vers l'hypertrophie.

L'étude successive au moyen du SEM (Henry \& Magniez, 1991), de la structure fine de l'organe copulateur d' $A$. hilgendorfii puis d'A. levanidovorum n. sp. nous a incités a réexaminer attentivement l'organe de l'espèce $A$. aquaticus d'Europe par le même moyen (Magniez \& Henry, 1970), afin d'effectuer des comparaisons morphologiques; elles seront évoquées plus loin.

Biogéographie. - L'aire de l'espèce est relativement réduite, incluse dans la grande aire du complexe "hilgendorfii" et à la fois continentale et insulaire (Fig. 18), mais on sait que les archipels côtiers de l'Extrême-Orient ont été très largement et à plusieurs reprises reliés au continent, en particulier au Pliocène et lors des régressions marines quaternaires. La répartition actuelle de l'espèce peut être expliquée par le jeu de ces oscillations paléogéographiques (Magniez, 1994).

Une question se pose néanmoins: l'île Hokkaido (au moins les bassins hydrographiques de son versant Nord) devrait logiquement être incluse dans cette aire. Or, les Aselles épigés de la région de Sapporo sont des A. hilgendorfii (cf. Matsumoto, 1963: 27). Les deux espèces pourraient donc y coexister, y compris sous forme de populations mixtes, comme au lac Bolon, et cela amène une question: les Aselles observés par Hilgendorf en 1874, près de la ville de Yedo étaient-ils des $A$. hilgendorfii ou des $A$. levanidovorum $n$. sp.?

La première éventualité implique que $A$. (A.) hilgendorfii mérite de conserver son nom spécifique. La seconde impliquerait par contre que tous les " $A$. hilgendorfii" cités dans la littérature postérieure à Bovallius (1886), et dont les dessins de pléopodes 
2 mâles ne montrent pas trace de canule ne sont pas de véritables hilgendorfii; c'est le cas en particulier des individus cités d'un puits à Pusan-City (Corée), dont l'organe copulateur ne comporte qu'une canule minuscule, pratiquement indécelable en microscopie optique (Henry \& Magniez, 1991). Il conviendrait dans ce cas de réhabiliter l'espèce $A$. (A.) nipponensis de Nicholls (1929)!

$A$. levanidovorum pouvant raisonnablement être considérée comme une espèce dérivée de la même souche qu'hilgendorfii, il est nécessaire d'envisager les événements paléogéographiques qui ont permis sa fondation et son extension actuelle. Hormis la possibilité d'une expansion récente liée aux activités anthropiques, le scénario suivant (Magniez, 1994), pourrait être proposé: (1) La forme-souche (très semblable à $A$. hilgendorfii) vit dans les eaux douces épigées continentales (bassin de l'Amour). (2) Une régression marine réunit l'île Sakhaline au continent, ce qui permet la colonisation de l'île par les Asellides. (3) L'île est à nouveau séparée du continent par une remontée du niveau marin (période interglaciaire ?): une population se trouve isolée du peuplement principal. Une évolution morphologique discrète modifie la structure fonctionnelle des pléopodes 2 mâles: il y a fondation d'une espèce nouvelle qui se répand dans l'île. (4) Occurrence d'une nouvelle phase géocratique (phase glaciaire ?): la nouvelle espèce étend son aire, à la fois vers le continent et vers des îles adjacentes (Nord d'Hokkaido, puis Kunashir). (5) Remontée du niveau marin (période thalassocratique Holocène): l'aire d'A. levanidovorum se trouve fragmentée en peuplements continentaux et insulaires, c'est la situation actuelle.

Une ultime question se pose: l'espèce levanidovorum a-t-elle, comme c'est le cas pour $A$. hilgendorfii, donné des formes stygobies dérivées dans les eaux souterraines de son aire et en particulier à Sakhaline? Des propections du milieu souterrain des trois îles concernées seraient indispensables pour conforter le scénario développé plus haut.

\section{Le complexe Asellus (Asellus) hilgendorfii}

Des collectes et observations complémentaires étant indispensables, nous ne nommerons pas prématuré- ment les variants régionaux du complexe "hilgendorfii" qui ne l'ont pas été par des auteurs antérieurs.

\section{D.1. Asellus (A.) hilgendorfii hilgendorfii} Bovallius, 1886 (Fig. 20)

Matériel examiné (ZMA Isop. 200 974). - Les stations japonaises sont décrites en détail par Broodbakker (1986); elles sont situées dans la partie $O$. de Honshu:

Stations: J.15, Yamaguchi-ken, Akiyoshi-cho, Akiyoshi-do, 18/10/1986, fossé avec eau courante: 14 adultes normalement pigmentés, avec des yeux bien développés et colorés, certains parasités par des larves d'Acanthocéphales. Le plus grand mâle atteint $8,5 \mathrm{~mm}$, la plus grande femelle $7,9 \mathrm{~mm}$.

J.25 et J.26, Yamaguchi-ken, Kununoki-cho, 20/10/1986, deux puits maçonnés obscurs. Dans le premier: 9 individus gris très clair avec yeux bien pigmentés, plus grand mâle de 7,5 et plus grande femelle de $4 \mathrm{~mm}$ (ovigère). Dans le second: env. 110 individus dont très nombreux juvéniles, gris très clair mais yeux normaux; plus grand mâle de 6,9 et plus grande femelle de $4,2 \mathrm{~mm}$.

J.37, Hiroshima-ken, Higashi-Hiroshima-shi, Befu Mitishita, 26/10/1986, puits maçonné obscur: 24 individus, adultes pour la plupart; teinte claire très variable, yeux pigmentés normaux. Les mâles atteignent 5,5 et les femelles $4,9 \mathrm{~mm}$.

J.41, Hiroshima-ken, Takehara-shi, Shinjo-cho, 26/10/1986, puits maçonné obscur: 21 individus de teinte claire mais variable, yeux normaux; mâles jusqu'à 5,0 et femelles jusqu'à $3,8 \mathrm{~mm}$. Une femelle anophtalme et dépigmentée de $5,9 \mathrm{~mm}$ de Phreatoasellus sp. dans le lot.

J.44, Hiroshima-ken, Kisa-cho, 28/10/1986, puits maçonné obscur: 9 jeunes légèrement pigmentés, avec yeux normaux, le plus grand atteint seulement $2,4 \mathrm{~mm}$. En plus une femelle à marsupium vide de $3,9 \mathrm{~mm}$ appartenant à une espèce anophtalme et dépigmentée indéterminable d'Asellus.

Remarques. - La forme-type japonaise a été redécrite en détail par Matsumoto (1963). Nous représentons à nouveau (Fig. 20), à titre de comparaison, l'endopodite copulateur.

\section{D.2. Asellus (A.) hilgendorfii ryukyuensis Uéno, 1938 (Fig. 21)}

Matériel examiné (ZMA Isop. 200 976). - Stations décrites en détail par Broodbakker (1986): J.59, Tamagusuku-son, S.O. Okinawa, 23/11/1986; Itokazu "Abuchira" (= grotte), ruisseau souterrain: environ 49 individus, dont de nombreux juvé- 
niles, fortement dépigmentés, de jaunâtres à presque blancs; yeux plus ou moins décolorés, parfois incolores, mais leur partie dioptrique subsiste; les mâles atteignent 5,8 et les femelles un peu plus de $4 \mathrm{~mm}$.

J.133, Yoron-to, Yoron-cho, 23/12/1986; Akasaki-do, cours de ruisseau souterrain à courant lent et flaques stagnantes: environ 30 adultes et subadultes, certains fragmentés et quelques jeunes de 1,8 a $2 \mathrm{~mm}$. Taille des mâles $<5,5 \mathrm{~mm}$, des femelles $<4 \mathrm{~mm}$. Presque toutes les femelles de 3,2 à $4 \mathrm{~mm}$ ont un marsupium vide, ce qui indique l'existence d'une reproduction massive au cours des mois précédant la capture. Les Aselles sont tous teintés, du gris-beige clair au blanc-jaunâtre, mais les yeux à 3 ommatidies sont toujours pigmentés de noir. Le tube contient également une demi-douzaine de gros Cyclopides blancs et un petit Oniscoïde dépigmenté avec 3 ommatidies colorées de chaque côté du céphalon.

J.135, Yoron-to, Yoron-cho, 23/12/1986; Yago, ruisseau souterrain: 1 jeune mâle de $2,8 \mathrm{~mm}$, teinte blanchâtre, mais yeux encore pigmentés.

Remarques. - La taille de cette sous-espèce insulaire (cf. Matsumoto, 1963) est plus faible que celle de la forme-type et surtout celle des formes nordiques. L'organe copulateur (Fig. 21) est très similaire à celui de la forme-type (Fig. 20), mis à part le processus calcariformis plus étroit basalement.

\section{D.3. Asellus (A.) hilgendorfii Bovallius, 1886; forme du Primorye et du bassin inférieur de l'Amour (Fig. 19)}

Matériel examiné. - Numéro de collection pour tout ce matériel asellidien: ZMA Isop. 200973.

Stations: L.1, “Southern Primorye, Popova Island, 09/08/ 1986, Maslov coll."': 8 adultes de teinte gris-clair, yeux pigmentés normaux. Taille max. des mâles: $6 \mathrm{~mm}$, des femelles: $5 \mathrm{~mm}$.

L.2, "Southern Primorye, Popova I., 11/08/1986, Maslov coll.": identiques aux précédents. Un mâle de $6,8 \mathrm{~mm}, 1$ femelle à marsupium vide de 5,2 et une femelle à oostégites réduits de $4,2 \mathrm{~mm}$.

L.3, "Southern Primorye, Popova I., Spring, 13/08/1978, Vshivkova coll.": 12 adultes très pigmentés et aux yeux noirs, dont 4 femelles ovigères. Taille max. des mâles: $5 \mathrm{~mm}$, des femelles: $4 \mathrm{~mm}$.

L.4, "Southern Primorye, Popova I., Spring, 05/08/1977, Vshivkova coll.": une douzaine d'individus, dont 3 jeunes de 2 a 2,5 mm, tous intensément pigmentés et normalement oculés. Taille max. des mâles: $6,6 \mathrm{~mm}$, des femelles: $6,2 \mathrm{~mm}$.

L.5, “Southern Primorye, Pelis I., Spring, 02/10/1983": au moins 200 individus, dont la moitié de jeunes; nombreuses femelles à poche incubatrice. Pigmentation intense, mais vari- able selon les spécimens; yeux normaux. Taille max. des mâles: $6 \mathrm{~mm}$, des femelles: $5,5 \mathrm{~mm}$. On observe que, dans toutes ces stations insulaires (L.1 à L.5), la période d'août-septembre est une saison de reproduction active.

L.6, "Vicinity of Vladivostok, Spring, 27/06/1988, Vshivkov coll.": 42 adultes et quelques jeunes, tous fortement pigmentés et normalement oculés. Taille max. des mâles: $7,8 \mathrm{~mm}$, des femelles: 5,6 mm. La taille moyenne des adultes est nettement supérieure à celle des populations insulaires précédentes.

L.7, "Primorye, Rudnaya River, 26/09/1984, Teslenko coll.": aucun Asellus dans ce tube, mais 5 petits ( $<2 \mathrm{~mm}$ ) Asellotes Janiroidea longilignes, anophtalmes et dépigmentés, ayant perdu presque tous leurs appendices et en mauvais état (Mackinia sp.); il n'est pas possible d'affirmer qu'il s'agit de $M$. continentalis Birstein \& Ljovuschkin, 1965, existant dans la région (ZMA Isop. 201 333).

L.9b, "Lower part of Amur River, Tributaries of Bolon lake, 12-14/05/1958, Levanidova coll.": 27 adultes normalement oculés et pigmentés (mais cette pigmentation est moins intense que celle des $A$. (A.) levanidovorum n. sp. présents [L.9a] dans ce prélèvement). Taille max. des mâles: $7,8 \mathrm{~mm}$, des femelles: 6,5 mm (les $A$. (A.) levanidovorum $\mathrm{n}$. sp. sont également beaucoup plus robustes). Dans cette station, la saison de reproduction ne semble pas encore commencée à la date de la capture.

L.10, "Spring in the vicinity of Khabarovsk town, 14/04/ 1963, Levanidova coll.": 1 grand mâle adulte de $7,5 \mathrm{~mm}$, fortement pigmenté et normalement oculé.

L.11, "Khabarovsk region, Komsomolsky reserve, Buylya spring, 27/07/1985, Makarchenko coll.": 23 adultes de pigmentation variable, du brun au gris-clair, yeux normalement pigmentés. Population robuste, taille max. des mâles: $9,3 \mathrm{~mm}$, des femelles: $6,8 \mathrm{~mm}$.

Remarques. - Les individus de cette région sont un peu plus robustes que la forme-type du Japon. La partie copulatrice de l'endopodite du pléopode II mâle (Fig. 19) est très semblable à celle des mâles japonais et des mâles du sud de la Corée, sur lesquels nous avons mis en évidence la canule (Henry \& Magniez, 1991); seul l'aspect du processus calcariformis diffère quelque peu (cf. Figs. 19 et 20).

\section{D.4. Asellus (A.) hilgendorfii, forme du Guangxi (Chine) (Fig. 22)}

Matériel examiné. - Station C.1, rivière souterraine de Xing'an (province de Guangxi, Chine). La localité ( $25^{\circ} 37^{\prime} \mathrm{N} 110^{\circ} 31^{\prime} \mathrm{E}$ ) est situé à environ $400 \mathrm{~km} \mathrm{N.O.} \mathrm{de} \mathrm{Canton} \mathrm{(Guangzhou);} \mathrm{matériel}$ récolté par B. et J. Lips le 25/08/1992 (ZMA Isop. 201 334). Spécimens dépigmentés mais parfaitement oculés: deux males adultes de 6,4 et 6,1 mm; une femelle adulte au repos génital de 

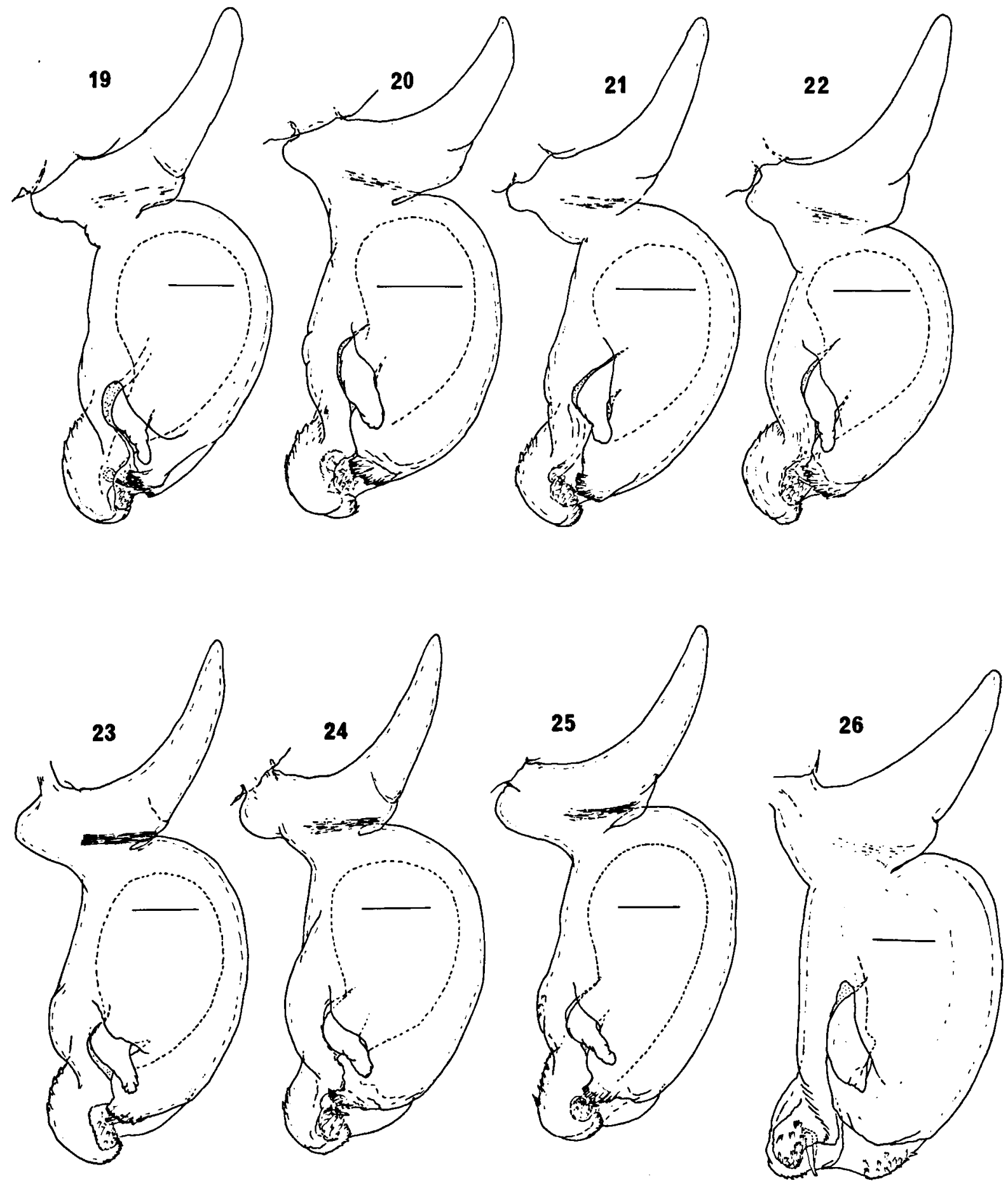

Figs. 19-26. Endopodites du pléopode 2 mâle droit, face sternale: 19, Asellus (Asellus) hilgendorfii de Popova Island; 20, A. (A.) hilgendorfii hilgendorfii du Japon; 21, $A$. (A.) hilgendorfii ryukyuensis d'Okinawa; 22, $A$. (A.) hilgendorfii forme de Chine méridionale; 23, $A$. (A.) hilgendorfii beringianus d'Anadyr; 24, A. (A.) hilgendorfii aff. beringianus du Kamchatka; 25, $A$. (A.) hilgendorfii tshaunensis du Chukotka; 26, Asellus (Arctasellus) birsteini du Chukotka; échelle de $50 \mu \mathrm{m}$ partout. 
$5,9 \mathrm{~mm}$ (la grande taille des oostégites non fonctionnels indique qu'elle a accompli très récemment une intermue parturielle); une femelle ovigère de 5,4 mm (environ 25 embryons dans le marsupium) et une femelle ovigère de $4,9 \mathrm{~mm}$ (environ 15 embryons dans le marsupium, à un stade de développement moins avancé que les précédents).

Remarques. - Tous les individus sont de teinte pâle, grisâtres. La pigmentation est légèrement plus intense sur la moitié antérieure du céphalon et forme également deux bandes longitudinales parasagittales sur le pléotelson (ces zones sont précisément plus fortement pigmentées chez les individus des populations épigées). Les yeux, formés d'au moins 3 ommatidies sont noirs, aussi pigmentés que ceux des populations épigées appartenant à la même espèce. Comme pour les autres Isopodes présentant des populations souterraines, on constate donc que la régression de la pigmentation somatique (face dorsale du céphalon, du péréion et du pléon) intervient avant celle de l'appareil dioptrique (Henry et al., 1986).

L'endopodite du pléopode 2 mâle (Fig. 22) est de type "hilgendorfii" dans son ensemble, cependant le lobe antérieur est plus volumineux et plus fortement écailleux qu'à l'habitude, ce qui masque davantage la canule. Le processus calcariformis est très semblable à celui de la forme-type japonaise.

\section{D.5. Asellus (A.) hilgendorfii, forme du lac Khanka}

Matériel examiné (ZMA Isop. 200 975). - Station L.8, “Khanka Lake, from stomach of a duck, 29/10/1979, Nechaev coll.": environ $\mathbf{4 5}$ individus adultes, mâles et femelles de teinte beige, aux yeux normalement développés et pigmentés. La taille ne semble pas excéder 7-8 $\mathrm{mm}$.

Remarques. - Les individus de ce lot ont été fortement déformés et altérés par le passage dans le tube digestif du canard et leur teinte s'y est sans doute éclaircie. L'aspect de l'exopodite des pléopodes 2 mâles semble légèrement différent, plus allongé que celui des $A$. hilgendorfii typiques, mais il ne s'agit peut-être que d'un artéfact lié à la macération. Pour la forme, nous isolons cet échantillon des précédents, mais il serait nécessaire d'observer cette structure sur du matériel en bon état pour justifier la séparation éventuelle de cette forme locale.

\section{D.6. Asellus (A.) hilgendorfii beringianus Levanidov, 1980 (Fig. 23, Pl. I, F)}

Matériel examiné (ZMA Isop. 200 977). - Stations: L.18, "Chukotka, Anadyr town, Lake near of airport, 12/06/1975, Chereshnev coll.": Lot non trié provenant de la station-type d'Asellus beringianus Levanidov, 1980: petits Bivalves, Gastéropodes sénestres et Planorbes, larves de Chironomides et autres Diptères, larves et fourreaux de Phryganes, Coléoptère, plus 83 Aselles adultes très polychromatiques (normalement pigmentés gris-beige, ou gris clair, ou blanchâtres avec céphalon et une zone médio-dorsale du corps teintés, ou encore totalement blanchâtres, mais tous conservant des yeux à 3 ommatidies parfaitement pigmentées de noir). Un individu est réfugié dans une coquille de Planorbe, seul le pléotelson avec les pléopodes respiratoires émergeant du péristome. Les mâles atteignent 8 et les femelles presque $7 \mathrm{~mm}$. Aucune de celles-ci ne présente de marsupium et aucun jeune n'est présent dans le prélèvement, mais les ovaires sont remplis de gros ovocytes, ce qui indique l'occurrence prochaine de la saison de reproduction (en juillet?).

L.31, "North part of Kamchatka Peninsula, Talovskoye Lake, 08/08/1980, Kurenkov coll.": 1 mâle adulte de $5,7 \mathrm{~mm}$, gris-beige clair et parfaitement oculé. La structure de l'organe copulateur (Fig. 24) montre qu'on peut le rapprocher d' $A$. ( $A$.) hilgendorfii beringianus (Fig. 23), les deux populations étant à inclure dans le complexe "hilgendorfii". Des prospections seraient indispensables pour préciser l'importance de ce peuplement du Kamchatka, géographiquement intermédiaire entre ceux du bassin de l'Amour et ceux de l'Anadyr.

\section{D.7. Asellus (A.) hilgendorfii tshaunensis Levanidov, 1980 (Fig. 25)}

Matériel examiné (ZMA Isop. 200 978). - Stations: L.20, "Chukotka, Pucheveem River, 07/07/1978, Makarchenko coll.": 2 mâles adultes de 8 et 7,2 mm, 1 femelle de 7,5 mm avec de nombreux oeufs et 1 femelle à oostégites réduits de $5,5 \mathrm{~mm}$.

L.21, "Chukotka, Pucheveem River, 11/07/1978, Makarchenko coll.": 3 mâles de 7; 5,5 et 4,5 mm; 1 femelle à marsupium avec une quinzaine de larves; 1 femelle à oostégites réduits de $4,5 \mathrm{~mm}$.

L.22, "Chukotka, Pucheveem River, 09/07/1978, Chereshnev coll.": 15 adultes, les mâles atteignant 8 et les femelles $7 \mathrm{~mm}$.

L.23, "Chukotka, Pucheveem River, 01/07/1978, Makarchenko coll.": 20 adultes dont 7 femelles à marsupium avec de très nombreux oeufs ou larves. Les mâles atteignent 9 et les 
femelles $8 \mathrm{~mm}$ (lot étudié par Levanidov, 1980 dans sa description d'A. tshaunensis).

L.24, "Chukotka, Pucheveem River, 01/07/1978, Chereshnev coll.": 1 mâle adulte de 10,2 mm.

L.25, "Chukotka, Chaun River, 22/07/1976, Atrashkevich coll.": lot-type ayant servi à la description d'Asellus tshaunensis Levanidov, 1980. Il en subsiste 34 paratypes mâles (taille max.: $9,1 \mathrm{~mm}$ ) et femelles (taille max.: $8 \mathrm{~mm}$ ), très polychromatiques et souillés de limon rouge. Présence de 21 femelles ovigères au marsupium bourré d'embryons.

L.26, "Chaun River basin, Lake, 26/06/1978, Chereshnev coll.": 23 adultes dont 5 couples en précopulation (mâle chevauchant la femelle à laquelle il est fixé par ses péréiopodes 4, dans l'attente de l'exuviation postérieure qui libérera les orifices génitaux femelles). Taille max. des mâles: $8,5 \mathrm{~mm}$, des femelles: $6,5 \mathrm{~mm}$.

L.27, "Magadan region, lower part of Chaun River, 23/06/ 1978, Makarchenko coll.": 91 adultes à pigmentation générale plus forte que dans les autres stations; carapace couverte d'Infusoires, ce qui montre qu'ils ont subi une longue période d'hivernage sans mues. $\mathrm{Ni}$ jeunes, ni femelles à poche incubatrice, mais uniquement des femelles aux ovaires gonflés de gros ovocytes, ce qui montre que la saison de reproduction n'a pas débuté à la date de capture. Taille max. des mâles jusqu'à $10 \mathrm{~mm}$; les femelles atteignent presque $9 \mathrm{~mm}$.

L.28, "Magadan region, Oyra River, 04/10/1979, Atrashkevich coll.": environ 55 adultes réduits à leur cuticule par altération des tissus internes (ce qui facilite l'observation de la canule en microscopie optique!), souvent fragmentés; teinte générale beige. Taille max. des mâles: environ $8 \mathrm{~mm}$, des femelles: environ 6,5 $\mathrm{mm}$ (aucune en phase de reproduction).

Remarques. - Pour les prélèvements L.20 à L.26, tous les individus sont pigmentées de façon très variable, en général du beige clair au beige foncé, la région céphalique souvent plus sombre que le reste du corps; les yeux sont toujours noirs et normalement développés ( 3 ommatidies). Une prédétermination libellée: “Asellus tshaunensis?" était jointe à chacun de ces tubes. On notera que la composition des prélèvements L.23, 25, 26, 27 indique l'existence, dans ces populations du bassin arctique des rivières Pucheveem et Chaun, d'une saison de reproduction très courte, mais très active: précopulation et ponte fin juin-début juillet; incubation en juillet; intermues parturielles terminées avant le début octobre (cf. L.28).

La représentation originale de l'endopodite du pléopode 2 mâle (Levanidov, 1980, p. 6, fig. 3G) est très sommaire. Notre Fig. 25 montre que cet organe est de forte taille, mais nettement de type "hilgendorfii" et qu'il convient d'inclure $A$. tshaunensis dans le complexe "Asellus hilgendorfii".

\section{D.8. Conclusions}

L'indépendance spécifique d'Asellus (Asellus) levanidovorum $\mathrm{n}$. sp. étant indiscutable, on constate que 1'“espèce"' Asellus (Asellus) hilgendorfii des auteurs (dont les espèces $A$. beringianus et $A$. tshaunensis semblent n'être que des variants géographiques de grande taille), constitue un immense ensemble de populations, disséminées sur un territoire s'étendant des rivages de l'océan Arctique (delta de la Léna, rivières Chaun et Pucheveem) jusqu'à la Chine méridionale (Guangxi) et comprenant les îles japonaises depuis Hokkaido jusqu'à Okinawa; cette aire pourrait inclure les bassins de quatre grands fleuves: Léna, Amour, Houang-Ho et même Yang-Tse-Kiang, ainsi que les bassins hydrographiques intermédiaires. Ces peuplements sont aujourd'hui génétiquement séparés et présentent déjà des différences morphologiques mineures, y compris au niveau de l'organe copulateur (taille générale de l'endopodite, forme de la partie basale du processus calcariformis, importance relative $\mathrm{du}$ lobe antérieur de la partie distale de l'endopodite, par exemple).

Compte tenu des différences climatiques régnant dans ces biotopes géographiquement très distants, on peut penser que des différences physiologiques considérables existent également entre peuplements arctiques et subtropicaux. Des expériences biologiques seraient indispensables pour savoir si ces peuplements sont toujours interfertiles, c'est-à-dire pour prouver ou non qu' $A$. hilgendorfii est encore une espèce biologique ou déjà un complexe d'espèces-soeurs. Dans l'attente d'un tel travail, nous proposons donc d'adopter une vision dynamique de ce complexe et de considérer $A$. ( $A$.) hilgendorfii come une super-espèce (superspecies), ce que, de toute manière, elle est condamnée à devenir, si elle ne l'est déjà, et incluant les différents variants cités plus haut dans le $\S \mathrm{D}$, plus Asellus hilgendorfii martynovi Birstein, 1947 des lacs du delta de la Léna.

Cette conclusion rejoint celle émise antérieurement par Botosaneanu (1988), dans son étude des Trichoptères de cette même aire périarctique (superespèce Micrasema gelidum McLachlan, Trichoptera) et nous devons citer ici une phrase fonda- 
mentale du travail de cet auteur (op. cit., 181): "Superspecies is a useful tool in studies of polytypic taxa with wide distribution and complex populational structure, ..." qui s'applique parfaitement au problème taxinomique posé par le complexe "hilgendorfii"'.

\section{E. Les relations avec Asellus (A.) aquaticus} (Linnaeus, 1758)

Cette espèce pigmentée et oculée est très largement répandue dans presque toute l'Europe continentale et insulaire plus l'Asie Mineure. Elle déborde même ces limites géographiques puisque nous avons récemment relevé sa présence dans l'île de Madère (Henry et al., 1994). Par ailleurs, nous avons pu examiner des spécimens parfaitement typiques, Asellus (Asellus) aquaticus aquaticus, provenant des eaux épigées de la région frontière entre Iran et Turkménistan, à l'Est de la mer Caspienne. Cependant, aucun individu de cette espèce n'a été observé dans les échantillons asiatiques étudiés ici et, en conformité avec l'opinion de Birstein (1951), il est vraisemblable que cette grande espèce n'existe pas en Extrême-Orient, sauf possibilités d'éventuels apports anthropiques locaux et récents. Il faut rappeler cependant qu' $A$. aquaticus reste l'espèce-type du genre Asellus sensu stricto et qu'une parenté étroite existe entre $A$. aquaticus et $\boldsymbol{A}$. hilgendorfii. Ce sont les deux espèces majeures du genre Asellus rénové et quelles que soient les révisions taxinomiques du futur, il est vraisemblable qu'elles resteront unies dans un même genre.

L'étude, au moyen du SEM, de la structure fine de l'organe copulateur d'A. aquaticus avait été entreprise depuis assez longtemps (Magniez \& Henry, 1970; Henry \& Magniez, 1977, 1991). Ces clichés ne montraient pas trace de canule. Tout au plus avaitil été remarqué que l'angle dièdre formé par le corps de l'endopodite et la masse du lobe antérieur, qui est allongé transversalement et très peu écailleux dans cette espèce, pouvait constituer éventuellement une gouttière spermatique efférente. La mise en évidence d'une petite canule chez $A$. $(A$.) hilgendorfii, sa présence chez l'espèce anophtalme $\boldsymbol{A}$.
(A.) primoryensis Henry \& Magniez, 1993 et enfin l'existence d'une canule de très grande taille chez. $A$. ( $A$.) levanidovorum $\mathrm{n}$. sp. ont donné à penser qu'il s'agissait d'une caractéristique morphologique constante dans le genre Asellus tout entier (qui se retrouve d'ailleurs dans les genres voisins d'Asellus). De nouveaux clichés SEM réalisés sur des $A$. aquaticus de Dijon ont montré que la canule existe également dans l'espèce européenne. Mais cette canule est beaucoup plus courte que celle d'hilgendorfii et profondément incluse dans la crypte formée par la spirale (très développée) du capitulum et l'angle distal-externe du lobe antérieur, si bien que son observation en microscopie optique est quasiment impossible. La membrane squamifère, qui enveloppe la base de la canule est également présente, mais de faible surface. Une orientation très précise de la platine porte-objet du SEM est par ailleurs nécessaire pour faire apparaître cet ensemble (PI. I, G). Sur les clichés antérieurs (Henry \& Magniez, 1977, pl. II/2, 1991, fig. 6), on devine l'existence d'une structure profonde entre la spirale du capitulum et la base du lobe antérieur, mais elle n'était pas, avec ces seuls documents, interprétable en tant que canule.

Ainsi, les clichés (Pl. I, A-G) et les schémas interprétatifs (Fig. 27) montrent que l'architecture générale des endopodites des pléopodes 2 mâles des espèces $\boldsymbol{A}$. aquaticus, $\boldsymbol{A}$. hilgendorfii et formes dérivées et $\boldsymbol{A}$. levanidovorum est bien identique, les mêmes éléments homologues (processus cylindriformis, lobe antérieur, capitulum, membrane squamifère et canule) étant présents, mais avec des dimensions et ornementations différentes. On peut donc conclure à l'existence d'un groupe évolutif naturel homogène parmi les Asellides eurasiatiques: les "vrais" Asellus. Il regroupe un ensemble occidental: $A$. aquaticus et tous ses variants stygophiles ou stygobies (sous-espèces ou formes locales énumérées par Sket, 1965), comme ceux de la grotte de Movile en Roumanie plus $A$. monticola Birstein, 1932, et un ensemble oriental: $A$. levanidovorum n. sp. plus $A$. hilgendorfii avec ses sous-espèces et espèces affines plus leur cortège d'espèces stygobies nippones (Henry et al., 1986), vraisemblablement dérivées d'hilgendorfii. 


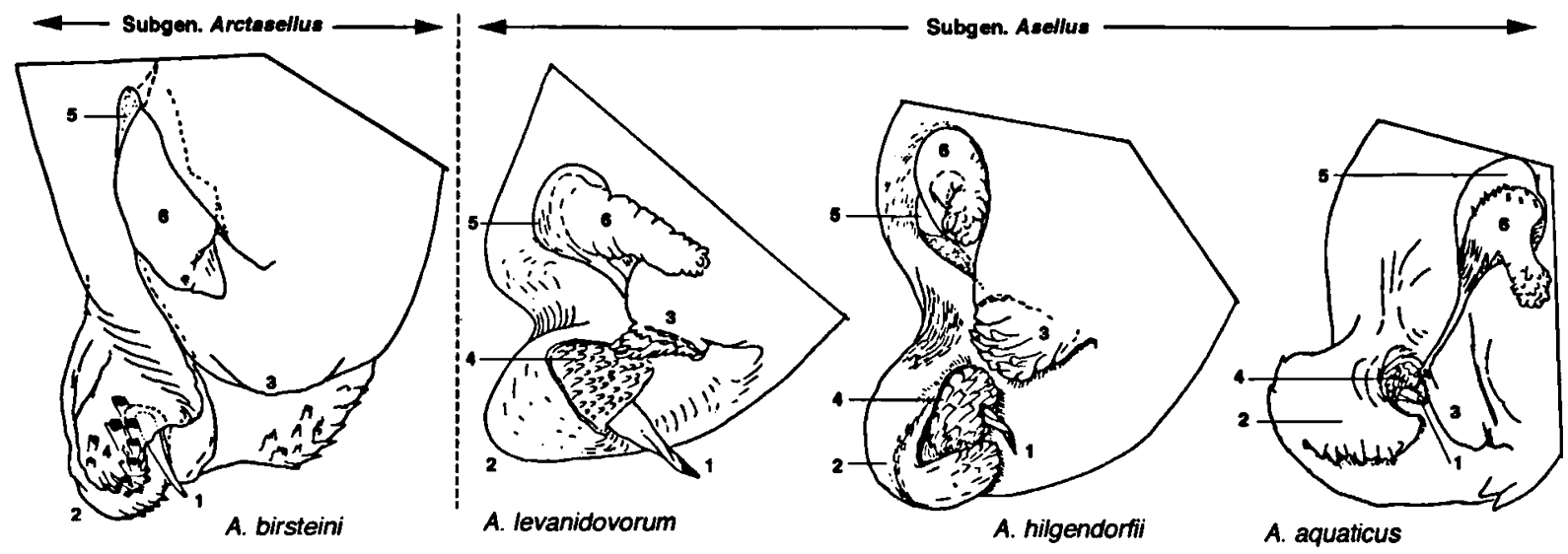

Fig. 27. Morphologie comparée de l'extrémité de l'endopodite du pléopode 2 mâle dans le genre Asellus, sous-genre Arctasellus et sousgenre Asellus; 1 = canule, 2 = capitulum, 3 = lobe antérieur, $4=$ membrane squamifère, 5 = orifice afférent, $6=$ processus cylindriformis; dessins d'après des clichés SEM.

\section{Les Asellus du groupe "latifrons" \\ (sous-genre Arctasellus nov. subgen.)}

Espèce-type: Asellus latifrons Birstein, 1947: 409.

En 1947, Birstein décrivit un Asellide très particulier, provenant des bassins de l'Ob et de l'Irtysh, jusqu'à des latitudes élevées $\left(64^{\circ} 55^{\prime} \mathrm{N}\right)$ : $A$. latifrons, caractérisé par un corps très élargi, par suite du grand développement des régions latérales du céphalon et des péréionites. La réduction des coxopodites à un anneau scléreux non mobile rend ceuxci totalement invisibles en vue dorsale. L'habitus est très semblable à celui des Lirceus américains, qui présentent le même trait morphologique et doit correspondre à une adaptation à la vie peltastique, mais la conformation de l'organe copulateur est nettement de type "Asellus" (présence du processus calcariformis et du "catch-lobe"), bien que la structure de détail de l'extrémité de l'endopodite soit assez différente de l'organisation typique constatée chez $A$. aquaticus ou $A$. hilgendorfii: la canule n'est pas insérée au fond d'une profonde crypte, mais elle émerge d'une aire squamifère pratiquement superficielle (cf. Fig. 27); le lobe antérieur est à peine marqué. La présence de 4 tiges seulement à la lame interne des maxillules permet d'attribuer cette forme au genre Asellus sensu stricto.

Par la suite, Bowman \& Holmquist (1975) décrivirent une espèce de l'Alaska très nettement ap- parentée à $A$. latifrons et Levanidov (1976) une troisième forme de la péninsule Chukotka, voisine des deux précédentes, ce qui montre l'existence d'une lignée arctique de l'ensemble "Asellus" qui a pu s'étendre jusqu'au continent américain à la faveur des périodes d'émersion du détroit de Béring. Les prélèvements étudiés ici comprennent des représentants de ces deux récentes espèces:

A. Asellus alaskensis Bowman \& Holmquist, 1975 (PI. I, H-I)

Matériel examiné (ZMA Isop. 200 979). - Station L.34, "Western Alaska, Kooper Bay [sans doute Hooper Bay?], 21/07/1976, Kontvimovichus coll.". Situation: région du delta de Yukon.

Lot capturé: 11 mâles adultes de 8,$1 ; 8,1 ; 8 ; 7,9 ; 7,7 ; 7,7 ; 7,6$; 7,$6 ; 7,3 ; 7,2$ et $6,9 \mathrm{~mm} ; 4$ femelles adultes à marsupium vide de 9,$5 ; 7,6 ; 7,3$ et $7 \mathrm{~mm} ; 3$ femelles ovigères de $7,1 \mathrm{~mm}$ (environ 20 pulli), $6,9 \mathrm{~mm}$ ( 4 jeunes prêts à éclore) et $6,8 \mathrm{~mm}$ (environ 30 oeufs). Une femelle à oostégites de repos génital de $7,2 \mathrm{~mm}$. La composition du lot montre que la population est encore en pleine saison de reproduction (ce que l'on observe en mars-avril pour les Asellus aquaticus d'Europe moyenne).

Remarques. - Les clichés SEM de l'extrémité de l'endopodite du pléopode 2 mâle (PI. I, H) confirment la représentation donnée par Bowman \& Holmquist (1975): le processus cylindriformis paraît aplati, atténué distalement et lisse, le capitulum est très peu développé, le lobe antérieur est 
presque nul, si bien que la vaste aire squamifère garnie d'écailles cuticulaires polydigitées et la fine canule membraneuse (Pl. I, I) ne sont aucunement abritées dans une crypte et protégées comme c'est le cas pour les Asellus typiques (groupe aquaticushilgendorfii $=$ sous-genre Asellus).

Les individus sont tous beige-clair et de plus souillés de limon ocre. Beaucoup contiennent des kystes de larves d'Acanthocéphales; une femelle contenait dans son marsupium une portion de Cestode adulte (scolex armé et muni de 4 ventouses plus plusieurs proglottis).

La situation de cette station montre que la répartition de l'espèce de l'Alaska s'étend largement au Sud de la zone des stations-types (lake No $^{\circ}$, lake Niglaktak, Inland Lake, Noatak et Selawik Lowlands), et sur une vaste zone comprenant le delta du Yukon, comme le montre Levanidov (1976) dans sa carte de répartition.

\section{B. Asellus birsteini Levanidov, 1976}

(Figs. 26-33)

Matériel examiné (ZMA Isop. 200 980). - Stations: L.12, "Khabarovsk rég., Tyoploye lake, 06/08/1974": 1 mâle de $6,7 \mathrm{~mm} ; 1$ mâle juvénile de $4,5 \mathrm{~mm}$; 3 femelles adultes de 8,7; 7,$9 ; 7,2 \mathrm{~mm}$, à oostégites de repos génital. Tous sont parfaitement oculés avec une teinte du corps jaunâtre.

L.13, "Khabarovsk rég., Tyoploye lake, 06/09/1974": 1 femelle adulte de $6,9 \mathrm{~mm}$ à oostégites réduits; tégument couvert de limon ocre.

L.14, "Chukotka, Tyoploye lake, 06/08/1974": 6 mâles adultes de 8,$2 ; 7,5 ; 7,3 ; 7,4 ; 7,0 ; 7,0$ (ce dernier en intramue); 3 mâles juvéniles de 4,$3 ; 4,2$ et $3,8 \mathrm{~mm} ; 6$ femelles adultes à oostégites réduits de 8,$0 ; 7,5 ; 7,2 ; 7,1 ; 7,1 ; 7,0 \mathrm{~mm} ; 2$ femelles juvéniles sans oostégites de 4,0 et $3,9 \mathrm{~mm}$. Individus blanchâtres avec légère teinte sur le céphalon et le pléotelson; yeux à 3 ommatidies parfaitement conformés et pigmentés de noir.

L.15, "Chukotka, Tyoploye lake, 06/08/1974": 1 jeune mâle de $4,8 \mathrm{~mm}$, pratiquement dépigmenté mais oculé.

L.16, Chukotka, Tyoploye lake, 06/08/1974, Vshivkova coll.": 3 mâles adultes de 8,1; 7,8 et 7,0 mm; 1 femelle adulte à oostégites réduits de $7,2 \mathrm{~mm} ; 1$ femelle immature sans costégites de 4,0 $\mathrm{mm}$ et 3 larves postmarsupiales à péréiopodes 7 rudimentaires de $2,1 \mathrm{~mm}$. Individus normalement oculés, mais corps jaunâtre et couvert de limon ferrugineux.

L.17, "Chukotka, Tyoploye lake, 06/09/1974": 8 mâles adultes de 8,$1 ; 8,1 ; 7,9 ; 7,4 ; 7,2 ; 7,1 ; 7,0$ et $4,6 \mathrm{~mm} ; 9$ femelles adultes à oostégites réduits de 8,$2 ; 8,1 ; 7,9 ; 7,8 ; 7,6 ; 7,5 ; 7,0 ; 6,9$ et $6,5 \mathrm{~mm} ; 2$ jeunes de 3,1 et $3,0 \mathrm{~mm}$, sexe indifférencié; plus les fragments de 5-6 individus de tailles variées. Tous ces individus présentent des yeux normaux ( 3 ommatidies, Fig. 28) et pig- mentés de noir, mais le polychromatisme du corps est important: les individus complètement pigmentés ont une livrée d'Aselles épigés normaux, tandis que les plus clairs sont jaunâtres, avec simplement une plage pigmentaire sur la moitié antérieure du céphalon (pouvant s'étendre sur la hampe des antennules et antennes; les yeux sont dans des plages claires, mais cernés d'un fin anneau pigmenté), une autre sur la région collaire entre tête et péréionite 1; sur le pléotelson, deux aires parasagittales en croissant, plus une plage impaire supra-anale; les extrémités des péréiopodes peuvent rester teintées. A fort grossissement les zones claires montrent néanmoins de fines traces colorées, ce qui indique que les chromatophores sont présents, mais quasiment dépourvus de granules pigmentaires.

L.19, "Chukotka, Lake no 3, 09/09/1974, Makarchenko coll.": 1 femelle adulte de $7,8 \mathrm{~mm}$; oostégites réduits; corps à peine pigmenté, sauf le céphalon, yeux normaux.

Remarques. - Les clichés SEM des organes copulateurs d' $\boldsymbol{A}$. alaskensis et $\boldsymbol{A}$. birsteini montrent que, bien que construits sur le même plan, ces organes sont quelque peu différents, ce qui peut justifier l'existence de deux espèces indépendantes. L'habitus onisciforme d' $A$. birsteini (Fig. 28) est très semblable à celui d' $A$. latifrons (Birstein, 1951, fig. 45) et à celui d'A. alaskensis (Bowman \& Holmquist, 1975, fig. 1).

Afin de compléter la description originale d' $\boldsymbol{A}$. birsteini, nous représentons ici l'endopodite du pléopode 2 mâle (Fig. 26), la région sternale d'un péréionite 4, montrant la réduction et l'ankylose du coxopodite (Fig. 29), ainsi que le pléopode 3 (Fig. 31), les exopodites des pléopodes 4 et 5 (Figs. 32 et 33) et la lame interne d'une des maxillules (Fig. 30), portant seulement 4 tiges ramifiées, caractère commun à tous les vrais Asellus, alors que le nombre de base est de 5 pour les Asellidae.

Note sur la distribution de l'espèce. - La présence d' $A$. birsteini dans des prélèvements de la région de Khabarovsk (L.12 + L.13) est étonnante, car elle traduirait un accroissement gigantesque de l'aire attribuée initialement à l'espèce. S'agit-il d'une expansion réelle, d'un peuplement récent d'origine anthropique ou d'une erreur d'étiquetage?

\section{Conséquences}

Les 3 espèces d'Asellus du groupe "latifrons" apparaissent aujourd'hui isolées les unes des autres 

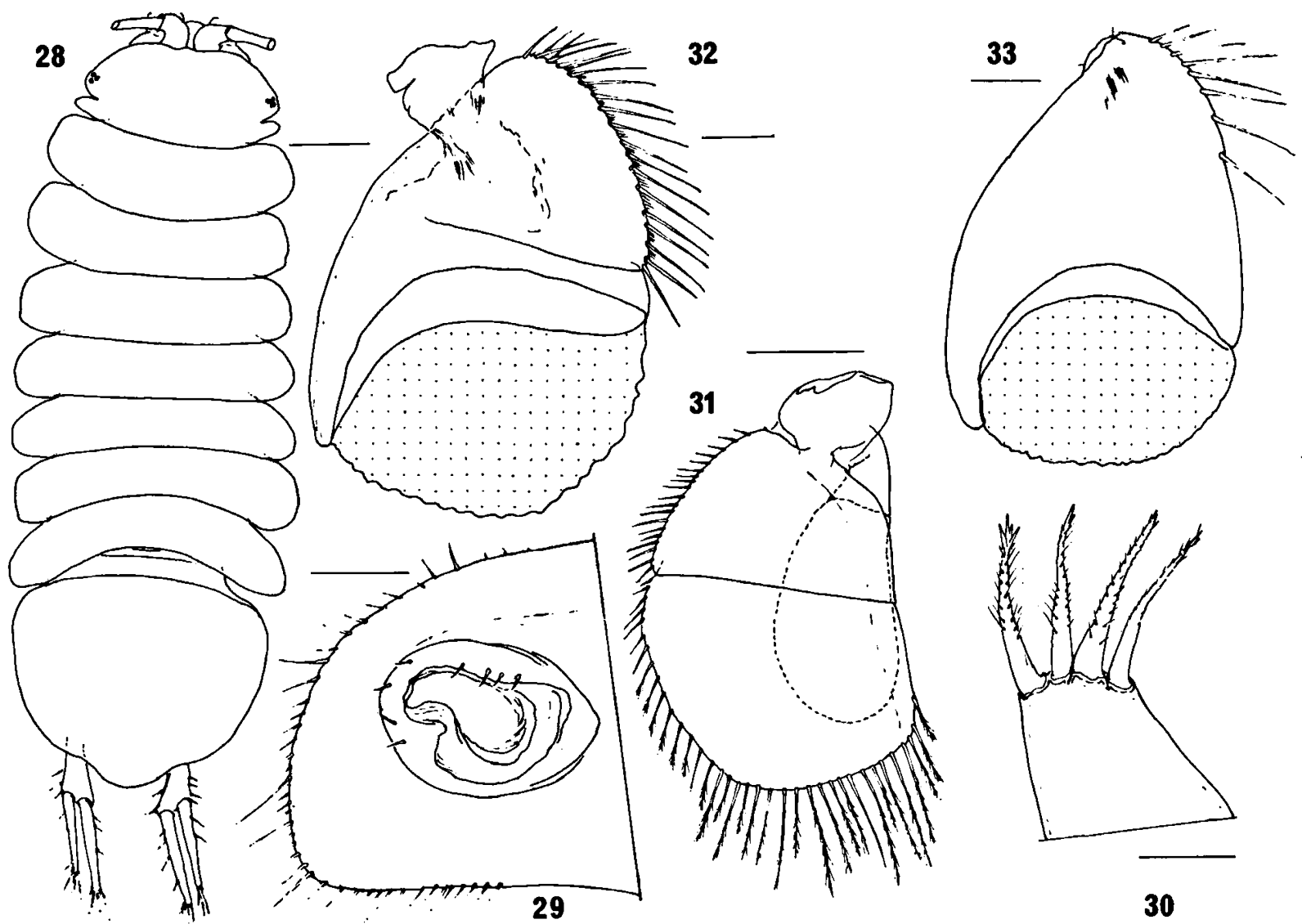

Figs. 28-33. Asellus (Asellus) birsteini: 28, habitus du mâle, échelle $=500 \mu \mathrm{m} ; 29$, face sternale gauche du péréionite 4 montrant le coxopodite annulaire, é $=200 \mu \mathrm{m} ; 30$, lame interne d'une maxille avec les 4 épines typiques d'Asellus, é $=50 \mu \mathrm{m} ; 31$, pléopode 3 droit, face sternale, $\hat{e}=500 \mu \mathrm{m} ; 32$, exopodite du pléopode 4 droit, face sternale, é $=200 \mu \mathrm{m} ; 33$, exopodite du pléopode 5 droit, face sternale, é $=200 \mu \mathrm{m}$.

sur un immense territoire sub-arctique et arctique. Elles constituent un groupe évolutif autonome au sein du genre Asellus, le sous-genre Arctasellus nov. subgen., dont on peut donner la diagnose formelle suivante:

- Présence des caractères généraux du genre Asellus E.L. Geoffroy, 1762.

- Coxopodites des péréiopodes réduits à un anneau uni au sternite.

- Canule de l'organe copulateur insérée superficiellement, membrane squamifère superficielle.

- Lobe antérieur de l'organe copulateur réduit ou inexistant.

Ces espèces se présentent apparemment comme des peuplements résiduels. Cependant, il est difficile de dire si cette situation correspond à la réalité, ou simplement à l'insuffisance des prospections des immenses territoires considérés. On peut également se demander si des espèces microphtalmes ou anophtalmes de ce groupe ne pourraient subsister dans les eaux souterraines de la partie tempérée du continent asiatique. L'exemple des Proasellus de l'Europe méridionale montre en effet que de telles espèces sont souvent très endémiques et seulement découvertes à la suite de prospections intensives.

\section{Conception actuelle du genre Asellus}

Dans deux publications antérieures (Henry \& Magniez, 1993; Magniez \& Henry, 1970), nous avons examiné l'évolution du contenu spécifique du genre Asellus E.L. Geoffroy. Ce genre est aujourd' hui expurgé de toutes les espèces non directement 
apparentées à l'espèce-type Asellus aquaticus (Linnaeus, 1758) et correspond mieux à la définition de la catégorie systématique genre. Sa définition s'appuie sur les principaux caractères suivants:

- Lobe interne des maxillules avec seulement 4 tiges ramifiées ( 5 tiges chez tous les autres Asellidae).

- Marge palmaire du propodite des péréiopodes 1 mâles avec un processus armé d'épines.

- Pléopodes 2 mâles présentant l'architecture de type Asellus ("Asellus pattern"), c'est-à-dire l'ensemble des caractères suivants: second article de l'exopodite muni d'un lobe tergal (catchlobe); endopodite avec une apophyse basale interne (ou processus calcariformis), orifice spermatique afférent de la vésicule interne pouvant être obturé par un processus digitiforme dit "processus cylindriformis", orifice spermatique efférent porté à l'extrémité d'une canule protégée par une membrane squamifère, présence d'un lobe antérieur et d'un capitulum terminal ...

Ce genre Asellus rénové comporte néanmoins deux groupes évolutifs d'espèces bien distincts l'un de l'autre:

(1) Groupe-type “aquaticus-hilgendorfii” (= sous-genre Asellus), caractérisé par ses coxopodites développés et libres, son organe copulateur à canule + membrane squamifère incluses dans une crypte par suite de l'existence d'un lobe antérieur et d'un capitulum fortement développés (Fig. 27), avec les espèces suivantes:

Asellus (Asellus) aquaticus (Linnaeus, 1758) et tous ses variants épigés, stygophiles et stygobies, y compris $A$. strinatii Chappuis, 1955 d'Asie Mineure; $A$. (A.) hilgendorfii Bovallius, 1886, et tous ses variants d'Extrême-Orient répertoriés plus haut; $A$. (A.) monticola Birstein, 1932, de Transcaucasie; $A$. (A.) epimeralis Birstein, 1947, du lac Baunt, Transbaiikalie; $A$. (A.) levanidovorum n. sp., ainsi que les espèces souterraines du Japon et du Primorye: $A$. (A.) hyugaensis Matsumoto, 1960 et $A$. (A.) kumaensis Matsumoto, 1960, de Kyûshû; $A$. (A.) shikokuensis Matsumoto, 1960, de Shikoku, A. (A.) amamiensis Matsumoto, 1961, de Ryû-Kyû, $A$. (A.) musashiensis Matsumoto, 1961 et. $A$. (A.) tamaensis Matsumoto, 1961, de Honshu; A. (A.) ezoensis Matsumoto, 1962, de Hokkaido; et enfin $A$. (A.) primoryensis Henry \& Magniez, 1993, de Sibérie sud-orientale, toutes appartenant manifestement à la lignée "hilgendorfii".

(2) Groupe "latifrons" à coxopodites réduits et endopodite copulateur avec membrane squamifère plus canule externes par suite de l'atrophie du lobe antérieur et du très faible développement du capitulum (Fig. 27). Ce groupe évolutif trispécifique pour l'instant devrait, pour des raisons de symétrie, prendre valeur de sous-genre (Arctasellus nov. subgen.) (= les Asellus de l'Arctique), comprenant:

Asellus (Arctasellus) latifrons Birstein, 1947, du bassin de l'Ob, Asellus (Arctasellus) alaskensis Bowman \& Holmquist, 1975, d'Alaska occidental et Asellus (Arctasellus) birsteini Levanidov, 1976, du Chukotka.

La connaissance du sous-genre Asellus pourrait être améliorée par une nouvelle étude, par des moyens modernes, des espèces asiatiques endémiques: Asellus monticola de Transcaucasie et Asellus epimeralis du lac Baunt (Transbaïkalie), par exemple, qui sont peut-être à l'origine des grandes espèces modernes expansives. Il est net dès maintenant que l'aire originelle des Asellus est extrême-orientale (périphérie de la Mer du Japon?), qu'A. aquaticus représente la forme la plus évoluée et que sa présence généralisée en Europe résulte d'une expansion récente et très rapide.

\section{La famille des Asellidae}

La connaissance des Asellidae du groupe "Asellus", considérés comme un ensemble supragénérique depuis les travaux de Matsumoto (1962), présente encore de nombreuses lacunes, par suite de l'immensité du territoire concerné: de la Transcaucasie et de la Chine subtropicale à l'Alaska et de celui-ci à la Californie (cf. Bowman, 1981). Les multiple études réalisées sur l'espèce $A$. (A.) aquaticus, la seule du groupe présente en Europe, n'apportent que peu de renseignements puisque cette forme moderne est allée proliférer et se diversifier récemment au dehors de l'aire asiatique propre à son groupe d'origine. Seules certaines aires restreintes (comme les îles japonaises) ont fait l'objet de prospections intensives, encouragées par la concentration d'espèces stygobies qui y existe. Or, 
FAMILLE DES ASELLIDAE

\begin{tabular}{|c|c|}
\hline \multicolumn{2}{|c|}{ Asellidae à pléopodes copulateurs de type "Asellus" (Asellus pattern) } \\
\hline Maxillules à 5 tiges distales & \multirow{2}{*}{$\begin{array}{l}\text { Maxillules à } 4 \text { tiges distales } \\
\text { (Genre Asellus E.L. Geoffroy) }\end{array}$} \\
\hline Palpe mandibulaire 3-articulé & \\
\hline $\begin{array}{l}\text { Gen. Mesoasellus Birstein } \\
\text { Gen. Phreatoasellus Matsumoto } \\
\text { Gen. Uenasellus Matsumoto } \\
\text { Gen. Calasellus Bowman } \\
\end{array}$ & $\begin{array}{l}\text { Canule dans une crypte } \\
\text { Subgen. Asellus } \\
\text { groupe "aquaticus/hilgendorfii" } \\
\text { (Eurasie) }\end{array}$ \\
\hline $\begin{array}{l}\text { Palpe mandibulaire réduit } \\
\text { Gen. Nipponasellus Matsumoto } \\
\text { Gen. Sibirasellus Henry \& Magniez }\end{array}$ & $\begin{array}{l}\text { Canule nue } \\
\text { Subgen. Arctasellus } \\
\text { groupe "latifrons" } \\
\text { (Sibérie arctique-Alaska) }\end{array}$ \\
\hline \multicolumn{2}{|c|}{$\begin{array}{l}\text { Autres lignées évolutives d' Asellidae: genres du lac Baïkal, de l'aire } \\
\text { périméditerranéenne, de la péninsule ibérique et de l'Amérique du Noro }\end{array}$} \\
\hline
\end{tabular}

Fig. 34. Situation du genre Asellus au sein de la famille Asellidae.

l'étude des lignées évolutives naturelles nécessite de prendre en compte à la fois les espèces épigées, les espèces microphtalmes et ensuite les espèces anophtalmes et dépigmentées qui en dérivent et la nature endémique de certaines fait qu'elle sont encore inconnues. Par ailleurs, le caractère national de beaucoup d'études les rend fragmentaires au plan biogéographique. Nous proposerons néanmoins ci-dessus un tableau (Fig. 34 ) pour tenter de définir, au moins provisoirement, les relations probables des différentes lignées évolutives du groupe "Asellus" au sens large (groupe qui a la dimension d'une sous-famille).

Au plan mondial, il semble de plus en plus net que la famille des Asellidae est un complexe hétérogène. Des ancêtres Asellotes marins ont conquis le milieu dulcicole continental à partir de points d'atterrissement privilégiés, éloignés les uns des autres (Ibérie, rivages de la Méditerranée et de la Paratéthys, Baïkal, périphérie de la mer du Japon, golfe du Mexique) et à des époques différentes. Ils ont donné des lignées qui se sont diversifiées et répandues avec plus ou moins de réussite pour chacune, profitant de l'évolution des réseaux hydrographiques. Il semble que, dans chacune de ces lignées, le passage des eaux marines aux eaux douces ait nécessité la réalisation d'un organe copulateur avec une vésicule interne (nécessité d'une protection osmotique du sperme en milieu à faible concentration ionique?). Cette réalisation s'est opérée de manière indépendante dans ces différentes lignées et si les endopodites des pléopodes 2 mâles dans les grands genres, tels au'Asellus, Proasellus, Bragasellus, montrent des analogies de structure, il semble s'agir de convergences fonctionnelles et non d'homologies (la partie copulatrice chez Asellus, et tout le groupe à "Asellus pattern", semble bien se réaliser à partir d'une expansion externe du premier article de l'endopodite, le second fournissant le processus calcariformis, alors que chez Proasellus et d'autres genres, c'est le second article qui développe ces structures spécialisées). La systématique des Asellidae doit donc s'appuyer en priorité, non pas sur les caractères somatiques (qui, le plus souvent sont, soit des caractères anciens, de valeur supra-familiale, soit des caractères d'adaptation circonstan- 


\section{Planche I}

A, Endopodite d'un pléopode 2 d'un mâle adulte Asellus (Asellus) hilgendorfii du Primorye. La petite canule est visible entre l'apex du lobe antérieur et celui du capitulum; échelle $=50 \mu \mathrm{m}$.

B, Le même pour $\boldsymbol{A}$. (Asellus) levanidovorum n. sp.: le sillon articulaire à la base du processus calcariformis et la longue canule sont très apparents; é $=100 \mu \mathrm{m}$.

C, Moitié distale du précédent, plus grossi: l'orifice spermatique afférent partiellement clos par le processus cylindriformis, le lobe antérieur écailleux, le capitulum formant un capuchon autour de la membrane squamifère et la canule sont visibles; un Infusoire phorétique sessile est fixé à l'entrée de la crypte; é $=50 \mu \mathrm{m}$.

$D$, Extrémité du précédent, lobe antérieur spatulé à marge distale entièrement garnie d'écailles cuticulaires; é $=40 \mu \mathrm{m}$.

$\mathrm{E}$, Canule et membrane squamifère du précédent; larges écailles cuticulaires à marge polydigitée et disposition tégulaire; ê $=10 \mu \mathrm{m}$. F, Moitié distale d'un endopodite de pléopode 2 de mâle d'A. (Asellus) hilgendorfii beringianus; organisation de type "'hilgendorfii"; sous cet angle, seule l'extrémité de la petite canule apparaît; é $=20 \mu \mathrm{m}$.

G. Extrémité d'endododite d' $A$. ( $A$.) aquaticus de Dijon: lobe antérieur très allongé transversalement et presque glabre, sauf dans l'angle distal-interne; capitulum hélicoïdal et armé de fortes épines; membrane squamifère minuscule et canule très courte, les deux totalement cachées dans la crypte fermée par la spire du capitulum et le lobe antérieur; $\hat{e}=25 \mu \mathrm{m}$.

H, Endopodite d'un mâle adulte d'Asellus (Arctasellus) alaskensis: processus cylindriformis court, lobe antérieur à peine marqué, extrémité de l'organe légèrement enroulée sur elle-même; é $=50 \mu \mathrm{m}$.

I, Extrémité du même, plus grossi: portion terminale correspondant au capitulum peu développée; une vaste membrane squamifère non abritée, avec des écailles longuement digitées, dont émerge une fine canule (c) du côté interne; é $=10 \mu \mathrm{m}$.

cielle au milieu), mair sur les caractères architecturaux des pléopodes sexuels, qui représentent des synapomorphies hautement significatives.

Ce sont les descendants actuels de chacune de ces lignées (espèces oculées de surface, plus les formes microphtalmes ou anophtalmes dérivées), qui devraient constituer les genres modernes des Asellidae. Les événements plio-quaternaires (par exemple phases glaciaires ayant décimé certaines lignées, débâcles post-glaciaires ayant permis des migrations d'espèces à très longue distance de leur centre de dispersion et la pullulation de formes expansives récentes), ajoutent des difficultés à la réalisation de cette enquête taxinomique.

\section{Bibliographie}

Birstein, J.A., 1932. Eine neue Art der Genus Asellus Geoffroy aus Transkaukasien. Zool. Anz., 98: 251-255.

Birstein, J.A., 1947. O vodyanykh oslikakh Sibiri (Water slaters of Siberia). Dokl. Akad. Nauk SSSR, 57(4): 409-412.

Birstein, J.A., 1951. Asellota. Faune de l'U.R.S.S., 7(5): 1-142 (Akad. Nauk. SSSR, Moscou) (en russe).

Botosaneanu, L., 1988. A superspecies, or Formenkreis, in caddisflies: Micrasema (superspecies gelidum) McLachlan (Trichoptera). Populational thinking versus Hennigian fundamentalism. Riv. Idrobiol., 27(2-3): 181-210.

Bovallius, C., 1886. Notes on the family Asellidae. Bih. K. svenska Vetensk. Akad. Handl., 11(15): 1-54.
Bowman, Th.E., 1981. Calasellus longus, a new genus and species of troglobitic asellid from Shaver Lake, California (Crustacea: Isopoda: Asellidae). Proc. biol. Soc. Wash., 94(3): 866-872.

Bowman, Th.E. \& Ch. Holmquist, 1975. Asellus (Asellus) alaskensis, n. sp., the first Alaskan Asellus, with remarks on its Asian affinities (Crustacea: Isopoda: Asellidae). Proc. biol. Soc. Wash., 88(7): 59-72.

Broodbakker, N.W., 1988. Ecology and distribution of groundwater Crustacea and freshwater Ostracoda in SW Honshu and the Nansei Islands, Japan. I. Groundwater Crustacea \& list of stations. Verslagen en technische Gegevens Inst. taxonom. Zoöl. Amsterdam, 53: i-ii, 1-21.

Derjavin, A.N., 1930. The freshwater Malacostraca of the Russian Far East. Russkii gidrobiol. Zh., 9: 1-8 (en russe, avec résumé en anglais).

Henry, J.-P., J.J. Lewis \& G. Magniez, 1986. Isopoda: Asellota: Aselloidea, Gnathostenetroidoidea, Stenetrioidea. In: L. Botosaneanu (ed.), Stygofauna mundi: 434-464 (E.J. Brill/Dr. W. Backhuys, Leiden).

Henry, J.-P. \& G. Magniez, 1970. Contribution à la systématique des Asellides (Crustacea Isopoda). Annls. Spéléol., 25(2): 335-367.

Henry, J.-P. \& G. Magniez, 1977. Observations sur Gallasellus heilyi (Legrand, 1956), représentant d'un nouveau genre d'Asellide souterrain de France. Bull. Soc. zool. Fr., 102(2): 215-222.

Henry, J.-P. \& G. Magniez, 1991. Quelques Asellotes stygobies et obscuricoles du Sud-Est de la Corée. Stygologia, 7(4): $217-$ 225.

Henry, J.-P. \& G. Magniez, 1993. Présence d'Asellides stygobies (Crustacea, Isopoda, Aselloidea) dans la région du Primorye, Sibérie sud-orientale. Bijdr. Dierk., 62(3): 179-191. 


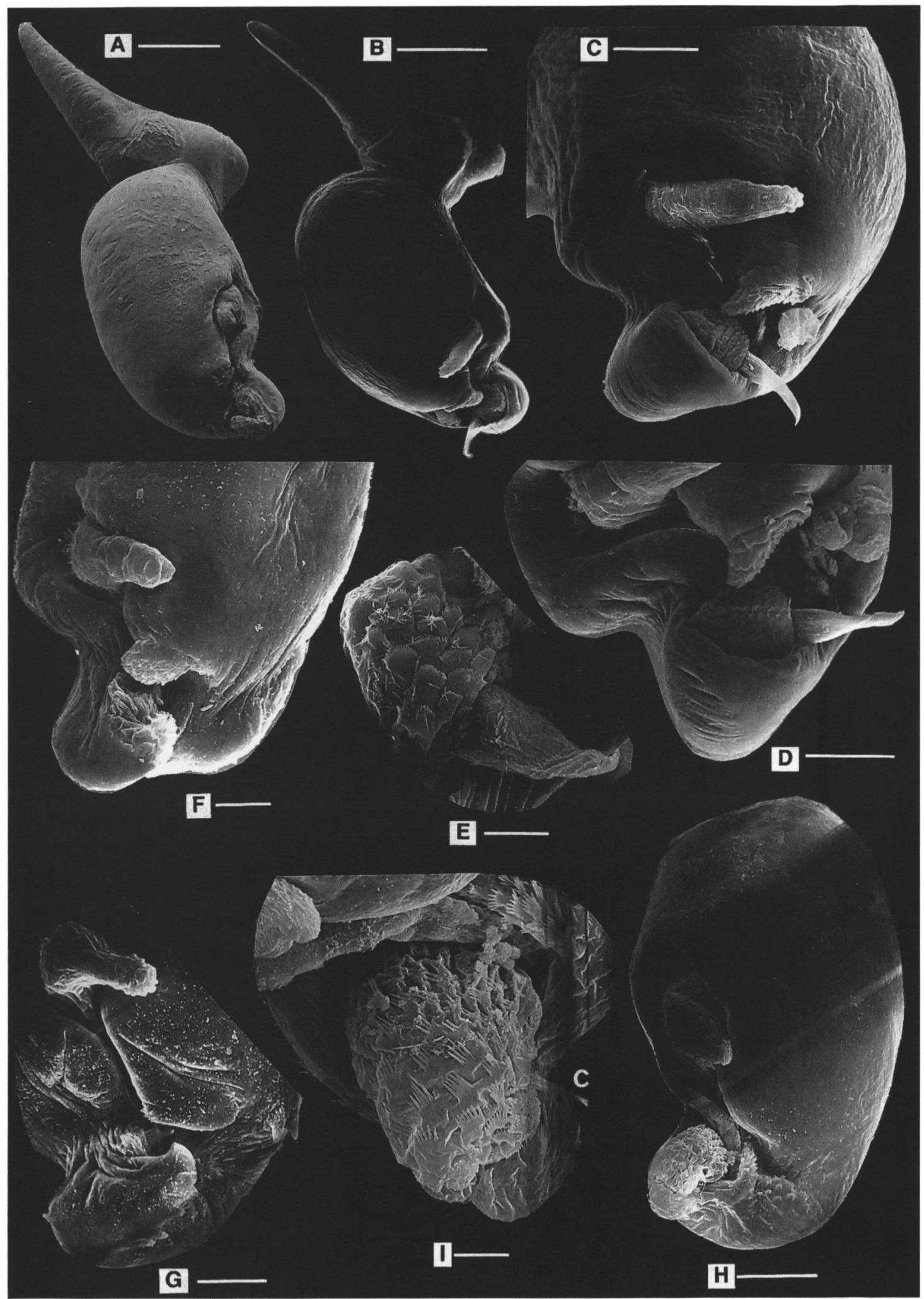


Henry, J.-P., G. Magniez, J.H. Stock \& J. Vermeulen, 1994. Les Asellidae (Crustacea Isopoda Aselloidea) de Madère et leur intérêt biogéographique. Mém. Biospéol., 21: 75-79.

Hilgendorf, F., 1874. Über eine Süsswasser-Assel. Mitth. dtsch. Ges. Natur-Völkerk. Ost-Asiens, 1(5): 39.

Hilgendorf, F., 1893. Bemerkungen über zwei Isopoden, die Japanische Süsswasser-Assel (Asellus hilgendorfii, Bov.), und eine neue Munna-Art. Sitzungsb. Gesells. naturf. Freunde, Berlin, 1: 1-3.

Kawamura, T., 1918. Freshwater biology of Japan: 1-237 (Tokyo) (en japonais).

Levanidov, V.Ya., 1976. A new species of the genus Asellus G. St. Hilaire from water bodies of the Chukotka Peninsula. Zool. Zh., 55(12): 1906-1908 (en russe).

Levanidov, V.Ya., 1980. Novyé vidy i rasnrostranenie vodyanykh oslikov Asellus s. str. (Isopoda, Asellidae) na severovostoke Azii. Fauna Presnykh Vod Dal'nego Vostoka: 13-23 (USSR Acad. Sci., Far East Sci. Branch, Vladivostok) (en russe).

Magniez, G., 1994. Vie insulaire des Asellidae et Stenasellidae en Extrême-Orient. Mém. Biospéol, 20(“1993”): 139-144.

Magniez, G. \& J.-P. Henry, 1970. Sur la structure des pléopodes copulateurs des Aselloidea (Crustacea Isopoda Asellota). C. r. hebd. Séanc. Acad. Sci. Paris, (D) 270: 93-95.

Matsumoto, K., 1962. Two new genera and a new subgenus of the family Asellidae of Japan. Annot. zool. Japon., 35(3): $162-169$.

Matsumoto, K., 1963. Studies on the subterranean Isopoda of Japan with notes on the well-water fauna of Japan (Part I). Studies on the subterranean Isopoda of Japan (No 1). Annls. Tokyo-to Lab. med. Sci., 13: 1-76.

Nicholls, G.E., 1929. A new species of Asellus (A. nipponensis) from Japan. Archs. Zool. expér. gén., 68 (Notes et Revue): 33-38.

Shen, C.-J., 1936. The freshwater isopods of Peiping. Bull. Fan Memorial Inst. Biol., (Zool.), 7: 1-31.

Sket, B., 1965. Taxonomische Problematik der Art Asellus aquaticus (L.) Rac. mit besonderer Rücksicht auf die Populationen Sloweniens. Razpr. Slov. Akad. Znan. Umet., Cl. IV Hist. Nat. Med., 8: 177-221.

Tattersall, W.M., 1921. Zoological results of a tour in the Far East. VII. Mysidacea, Tanaidacea and Isopoda. Mem. Asiat. Soc. Bengal, 6: 403-433, pls. 15-17.

Uéno, M., 1938. Asellus from the Ryukyu Islands. Trans. nat. Hist. Soc. Formosa, 28: 264-268.

Wei, C.T., 1970. Order Isopoda of the Hangchow Region. Communist China Sci. Abstracts, 2: 177.

Reçu le 6 juin 1994 